\title{
Accelerated Expansion and Structure Formation with a Single Scalar Field
}

\author{
Tonatiuh Matos ${ }^{\mathrm{a}, *}$, Juan Magaña ${ }^{\mathrm{b}}$ and Abril Suárez ${ }^{\mathrm{a}}$ \\ ${ }^{a}$ Departamento de Física, Centro de Investigación y de Estudios Avanzados del IPN, A.P. 14-740, 07000 México D.F., \\ México; ${ }^{b}$ Instituto de Astronomía,Universidad Nacional Autónoma de México, AP 70-543, 04510 D.F., México
}

\begin{abstract}
In this work we calculate and simulate the different ingredients needed to obtain the matter density profile of the large-scale structure we observe now days in a qualitative way. This work begins assuming that the seeds of the early universe were small fluctuations that lived inside a homogeneous FLRW background, and which are studied using the Linear Perturbation Theory, these fluctuations then grew because of gravitational instability, in this way giving birth to what we see today: galaxies, clusters of galaxies, etc. The key difference of this work compared with other works lays in the fact that we introduce $\Phi^{2}$ as a scalar field candidate to dark matter, and as one of the main ingredients needed to obtain the large-scale structure we see today. We then solve the obtained set of equations like a dynamical system in which we introduce new dimensionless parameters that describe the cosmology of the different studied quantities, this shows us that both; the gravitational potential and the fluctuations on the scalar field are the main ingredients to the spectrum of the density contrast, the last one being responsable of the description of matter distribution we observe these days. Also, we have developed the non linear regime with the sphericall collapse formalism in order to compute the parameters of virialized structure with our hypothesis. Finally, we show how the quantum effects of the same scalar field can explain the value of the cosmological constant.
\end{abstract}

Keywords: Scalar field, sphericall collapse, structure formation.

\section{INTRODUCTION}

One of the most fundamental problems in modern Cosmology is to know the nature of dark matter. The standard model of cosmological structure formation in the Universe is Lambda cold dark matter ( $\Lambda \mathrm{CDM})$. Most of all cosmological observations support it, like the large-scale structure of the Universe and the spectrum of CMB, but this paradigm has some problems at galactic scales, like the central densities in LSB galaxies and the number of satellite galaxies.

The Cosmic Microwave Background radiation (CMB) is one of the main proves about the homogeneous and isotropic models in the Big Bang theory. The anisotropies seen in the CMB are related with small perturbations that are found in a perfectly smooth background, and are believed to be the seeds in the formation of galaxies and large-scale structure in the Universe. We then assume that in the past existed small deviations in such homogeneties in our Universe.

There are several alternative models propposed in order to solve the problems of $\Lambda \mathrm{CDM}$, some of these models are: warm dark matter, auto-interacting dark matter, $f(R)$ Gravity (see [1] for an excellent introduction to this field) and Modified Gravity (MoG) [2], being these two last theories very close related with the Scalar-Tensor-Theories of gravity (STTG). At this point it is worth to remark that the scalar field appearing in these theories can be interpreted as a

*Address correspondence to this author at the Departamento de Física, Centro de Investigación y de Estudios Avanzados del IPN, A.P. 14-740, 07000 México D.F., México; E-mail: tmatos@fis.cinvestav.mx source or as part of the curvature. In this paper we will adopt a minimally coupled scalar field, with a $\Phi^{2}$ scalar field potential, minimally coupled to matter, being this the most simple version of the STTG, called the Scalar Field Dark Matter (SFDM) model [3-12]. We study SFDM in order to investigate if this model is able to explain the formation of structure. SFDM supposes that dark matter is a minimally coupled real scalar field with a scalar potential that interacts only gravitationally with the rest of the matter. The motivation of this model is that it be behaves like CDM and it could solve many problems of the standard model in addition to which it could form gravitational structures.

For cosmological models that involve SFDM, it is usually assumed that the SF does not show density fluctuations on cluster scales or below. This is because in Linear Perturbation Theory, the mass of the SF is very small and because of this, it does not feel small fluctuations. If the scene changes, and the mass of the SF does feel the fluctuations, we then also have to take into account nonlinear perturbations, that could modify the evolution of the perturbations in the dark matter, and this affect the evolution in structure formation.

The main objective in introducing a SF model as dark matter is to assume that such dark matter is a scalar field that involves a minimally coupled potential of the form

$$
V(\Phi)=\frac{1}{2} m^{2} \Phi^{2}
$$

where the mass of the scalar field $\Phi$ is defined as $m_{\Phi}=\ddot{V}_{\Phi=0}$. and the dark matter is affected by radiation only indirectly, through the gravitational potential. 
If we want to study these anisotropies we need to know how the perturbations that act upon the dark matter evolve. This can give us the possibility of giving the energy density necessary to obtain the amount of matter observed in galaxies an required to study the rotation curves in stars, as to give the correct distribution of dark matter in them.

The evolution of these fluctuations with specified magnitude are defined by several fundamental parameters, like: the cosmic density $\Phi$, the cosmological constant $\Lambda$ and the relative contributions of radiation, and the densities of dark and visible matter in the universe. We then define the relative fluctuations on the mass density as $\delta \equiv \frac{\delta \rho}{\rho}$ where $\rho$ is the mass density in the Universe.

We develop the theory and the numerical simulations to obtain such spectrums, based on all the facts mentioned earlier, and making reference to the non-perturbed model (without fluctuations) as the background from which we evolve the small perturbations to what we see today.

\section{THE STATISTIC OF A BEC}

In this section we review the condensation of an ideal Bose gas of $N$ particles of mass $m$ contained in a volume $V$ with temperature $T$ and with only a portion $\rho_{0}$ of the system in the ground state. In order to see that and to be self contained, let us start from its grand partition function $Q$, which is given by

$$
Q(z, V, T)=\prod_{p} \frac{1}{1-z e^{-\beta \varepsilon_{p}}},
$$

where the fugacity $z=e^{\beta u}$ is defined in terms of the chemical potential $\mu$ and $\beta=1 / T$. In this paper we use the fundamental constants $\hbar=c=k_{B}=1$.

Then, the state equation for an ideal Bose gas is

$$
\frac{P V}{T}=\log Q=-\sum_{p} \log \left(1-z e^{-\beta \varepsilon_{p}}\right) .
$$

Thus the grand partition function directly gives the pressure $\mathrm{P}$ as a function of $z, V$, and $T$.

The particle number $N$ and the internal energy $U$ are

$$
\begin{aligned}
& N=z \frac{\partial}{\partial z} \log Q=\sum_{p} \frac{z e^{-\beta \varepsilon_{p}}}{1-z e^{-\beta \varepsilon_{p}}}, \\
& U=-\frac{\partial}{\partial \beta} \log Q=\sum_{p} \frac{\varepsilon_{p} z e^{-\beta \varepsilon_{p}}}{1-z e^{-\beta \varepsilon_{p}}}, .
\end{aligned}
$$

Where $\varepsilon_{p}$ is the single-particle energy with momentum $p$ and the average occupation numbers $\left\langle n_{p}>\right.$ are given by

$$
<n_{p}>=\frac{z e^{-\beta \varepsilon_{p}}}{1-z e^{-\beta \varepsilon_{p}}},
$$

which satisfy the conditions

$$
\begin{gathered}
N=\sum_{p}<n_{p}>, \\
U=\sum_{p} \varepsilon_{p}<n_{p}>.
\end{gathered}
$$

Now we let $V \rightarrow 0$ taking the limit of continuity, and replace sums over $\mathbf{p}$ by integrals over $\mathbf{p}$, we the obtain the following equation of state.

$$
\begin{gathered}
\frac{P V}{T}=-\frac{2 V}{(2 \pi)^{2}} \int_{0}^{\infty} d p p^{2} \log \left(1-z e^{-\beta p^{2} / 2 m}\right)-\log (1-z), \\
N=\frac{2 V}{(2 \pi)^{2}} \int_{0}^{\infty} d p p^{2} \frac{z e^{-\beta p^{2} / 2 m}}{1-z e^{-\beta p^{2} / 2 m}}+\frac{z}{1-z} .
\end{gathered}
$$

These equations can be written into the equivalent form

$$
\begin{aligned}
& \frac{P V}{T}=\frac{V}{\lambda^{3}} g_{5 / 2}(z)-\log (1-z), \\
N= & \frac{V}{\lambda^{3}} g_{3 / 2}(z)+\frac{z}{1-z},
\end{aligned}
$$

where $\lambda=\sqrt{2 \pi / m T}$ is the thermal wavelength, and

$$
\begin{aligned}
& g_{5 / 2}(z)=-\frac{4}{\sqrt{\pi}} \int_{0}^{\infty} d x^{2} \log \left(1-z e^{-\beta x^{2}}\right), \\
& g_{3 / 2}(z)=z \frac{\partial}{\partial z} g_{5 / 2}(z) .
\end{aligned}
$$

Therefore internal energy is found from the formulas (2) and (4)

$$
U=\frac{3}{2} \frac{T V}{\lambda^{3}} g_{5 / 2}(z)
$$

and a consequence the relation $\mathrm{U}=3 / 2 \mathrm{PV}$ is fulfilled.

From equation (5) we see that

$$
<n_{0}>=\frac{z}{1-z},
$$

which is the average occupation number for a single particle with occupation level $p=0$. Equation (10) can also be written as

$$
\lambda^{3} \frac{<n_{0}>}{V}=\lambda^{3} \frac{N}{V}-g_{3 / 2}(z)
$$

This equation tell us that $\frac{\left\langle n_{0}\right\rangle}{V}>0$ and therefore the temperature and the specific volume are such that $\lambda^{3} \frac{N}{V}>g_{3 / 2}(z)$. This means that a finite fraction of the particles will be in the ground state with $p=0$, i.e., the Bose gas condensates. In the region of condensation, the fugacity $z: 1$ and the functions $g(z)$ goes to the Riemann $\zeta$ function $g_{l}(z) \rightarrow \zeta(l)$. 
And the thermodynamical surface which separates the condensation region from the rest of the $P-V-T$ space, is given by

$$
\lambda_{c}^{3} \frac{N}{V}=g_{3 / 2}(1)=2.612,
$$

thus $\lambda_{c}$ can be interpreted as the value for which the thermal wavelength is of the same order of magnitude as the average interparticle separation. Equation (15) defines the critical temperature for which the Bose condensate forms. This temperature is given by

$$
T_{c}=\frac{2 \pi}{m_{\Phi}^{5 / 3}}\left(\frac{\rho}{g_{3 / 2}(1)}\right)^{\frac{2}{3}},
$$

where $\rho=m_{\Phi} N / V$ is the density of the Bose gas. At constant temperature, equation (16) defines a critical density

$$
\rho_{c}=\frac{m_{\Phi} g_{3 / 2}(z)}{\lambda^{3}} .
$$

Thus, the region of condensation of the Boson gas is determined by $T<T_{c}$ or $\rho>\rho_{c}$

After the Bose gas condensates most of the bosons lie in the ground state, the scalar field starts to oscillate around the minimal of its potential and the scalar field starts to behave as dust [13]. Thus, after the scalar field decouples from the rest of the matter, the temperature of the BEC's goes like

$$
T_{B E C}=T_{B E C}^{(0)}\left(\frac{a_{0}}{a}\right)^{2}
$$

where $T_{B E C}^{(0)}$ is the actual temperature of the BEC's and $a_{0}=1$ the value of the scale factor at the present.

In the same way, as the BEC's behave as matter, their density goes like $\rho_{B E C}=\rho_{B E C}^{(0)} / a^{3}$, where $\rho_{B E C}^{(0)}$ is the actual matter content of BEC's in the universe. With this result equation (16) can be also transformed into the form

$$
\begin{aligned}
& T_{c}=\frac{2 \pi}{m_{\Phi}^{5 / 3}}\left(\frac{\Omega_{B E C}^{(0)} \rho_{c r i t}}{\zeta(3 / 2)}\right)^{\frac{2}{3}} \frac{1}{a^{2}}, \\
& =6.2 \times 10^{-31} \frac{\left(\Omega_{B E C}^{(0)} h^{2}\right)^{2 / 3}}{\left(m_{\Phi} / G e V\right)^{5 / 3}} \frac{1}{a^{2}} G e V,
\end{aligned}
$$

where $\Omega_{B E C}^{(0)}$ is the actual rate of BEC's and $\rho_{\text {crit }}$ is the critical density of the universe.

If the actual standard model of particles scheme could be extended to higher temperatures, we have to expect that the scalar field which forms the BEC, interacts with the rest of the particles to a temperature over some temperature $T_{s}$. Because the physics of the elemental particles is well known till temperatures like $\mathrm{GeV}$, we do not expect that so an exotic particles as these scalar fields appear under temperatures like $\mathrm{TeV}$. Here we have two possibilities, the first one is that the scalar field has never had interaction with the rest of the particles and it evolves independently from the rest of the fields, with only a gravitational interaction. In this case the scalar field condensate from the beginning of the universe. The second possibility is that in the early universe the scalar field lived unify with the rest of the particles in a thermal bad and in some moment in its evolution, separates from the interaction. If this is the case let us suppose here that the scalar field which forms the BEC decouples from the rest of the matter at a temperature over $\mathrm{TeV}$. Under this temperature, this scalar field has almost no interaction with the rest of the matter. If we expect that this scalar field forms a BEC, its critical temperature must be lower than the temperature of the scalar field decoupling. This fact gives us an upper bound of the mass $m_{\Phi}$ of the scalar field

$$
m_{\Phi}<10^{-17} e V
$$

On the other hand, from numerical simulations [14] we know that scalar fields form gravitationally bounded objects with a critical mass given by

$$
M_{c r i t} \sim \tilde{m} \frac{m_{p l}^{2}}{m_{\Phi}},
$$

where $m_{p l}$ is the Planck mass and $\tilde{m}$ is a factor such that $\tilde{m} \approx 0.6$ for complex scalar fields (boson stars) and real scalar fields (oscillatons). With the value given in (21), the scalar field can form a gravitationally bounded BEC with a critical mass given by

$$
\begin{aligned}
& M_{c r i t}>1.491 \times 10^{64} \mathrm{GeV}, \\
& =2.658 \times 10^{40} \mathrm{gr}, \\
& =13.36 \times 10^{6} M_{\mathrm{e}} .
\end{aligned}
$$

This is an interesting result, if there exist a scalar field and plays any role in the universe at this moment, this scalar field must have a mass lower than the mass given in (21) and they are forming gravitationally bounded BEC's with masses around the mass given in [15].

\section{SELF-GRAVITATING BEC}

In this section we give some general features of the gravitational collapse of the BEC, we pretend only to show a generic behavior of any self-gravitating BEC. The BEC cosmology have been studied by [16] and many numerical simulations of this collapse are given in $[15,17,18]$ and by besides. [19] found that BEC in the ground state are very stable under different initial conditions. After the Bose gas condensates the gravitational force makes the gas collapse and form self-gravitating objects. Let us suppose that the halo is spherically symmetric, which could not be to far from the reality. In that case, the space-time metric reads

$$
d s^{2}=-e^{2 v} d t^{2}+\frac{d r^{2}}{1-\frac{2 M G}{r}}+r^{2} d \Omega^{2},
$$


where the function $v=v(r)$ is essentially the Newtonian potential and $M=M(r)$ is the mass function given by

$$
\begin{aligned}
& M=4 \pi \int \rho r^{2} d r \\
& \frac{d v}{d r}=G \frac{M+4 \pi r^{3}}{r^{2}\left(1-\frac{2 M G}{r}\right)} .
\end{aligned}
$$

The Einstein field equations reduce to equations (27) and the Oppenheimer-Volkov equation

$$
\frac{d P}{d r}=-G \frac{(P+\rho)\left(M+4 \pi r^{3}\right)}{r^{2}\left(1-\frac{2 M G}{r}\right)} .
$$

Let us focus in the case when the gas is far from forming a black hole. In that case we suppose that $2 M G<<r$ and equation (28) reduces to

$$
\frac{d P}{d r}=-4 \pi G r P(P+\rho) .
$$

The state equation can be obtained from the equation $P V=2 / 3 U,(10)$ and (12). Combining all equations we obtain that

$$
\begin{gathered}
P=\frac{2 \pi}{m_{\Phi}^{8 / 3}} \frac{g_{5 / 2}(z)}{g_{3 / 2}(z)^{5 / 3}}\left(\rho-\rho_{0}\right)^{5 / 3}, \\
=\omega\left(\rho-\rho_{0}\right)^{5 / 3},
\end{gathered}
$$

where $\omega$ is the constant

$$
\omega=\frac{2 \pi}{m_{\Phi}^{8 / 3}} \frac{g_{5 / 2}(z)}{g_{3 / 2}(z)^{5 / 3}},
$$

and $\rho_{0}=m_{\Phi}<n_{0}>/ V$ is the mean density of the particles in the ground state. Thus, the Oppenheimer-Volkov equation (29) transforms into

$$
\frac{d \rho}{d r}=-\frac{12}{5} \pi G r\left(\rho-\rho_{0}\right)\left(\omega\left(\rho-\rho_{0}\right)^{5 / 3}+\rho\right) .
$$

This differential equation can be easily numerically solved. Nevertheless, we have two interesting limits of equation (33). First suppose that the $\omega$ constant is small such that $P<<$. This situation occurs for big scalar field masses $m_{\Phi}: m_{\text {Planck }}$. In that case, the equation (33) contains an analytical solutions given by

$$
\rho(r)=\frac{\rho_{0}}{1-\left(1-\frac{\rho_{0}}{\rho(0)}\right) e^{-\frac{6}{5} \pi G \rho_{0} r^{2}} .}
$$

Observe that when $r \dagger \infty$, the function $\rho(r) \dagger \rho_{0}$. The function changes dramatically for different values of $r_{0}$. If $r_{0}>1$, the density $\rho(r)$ decreases, but if $r_{0}<1$ the density increases. The behavior of the density is shown in Fig. (1).
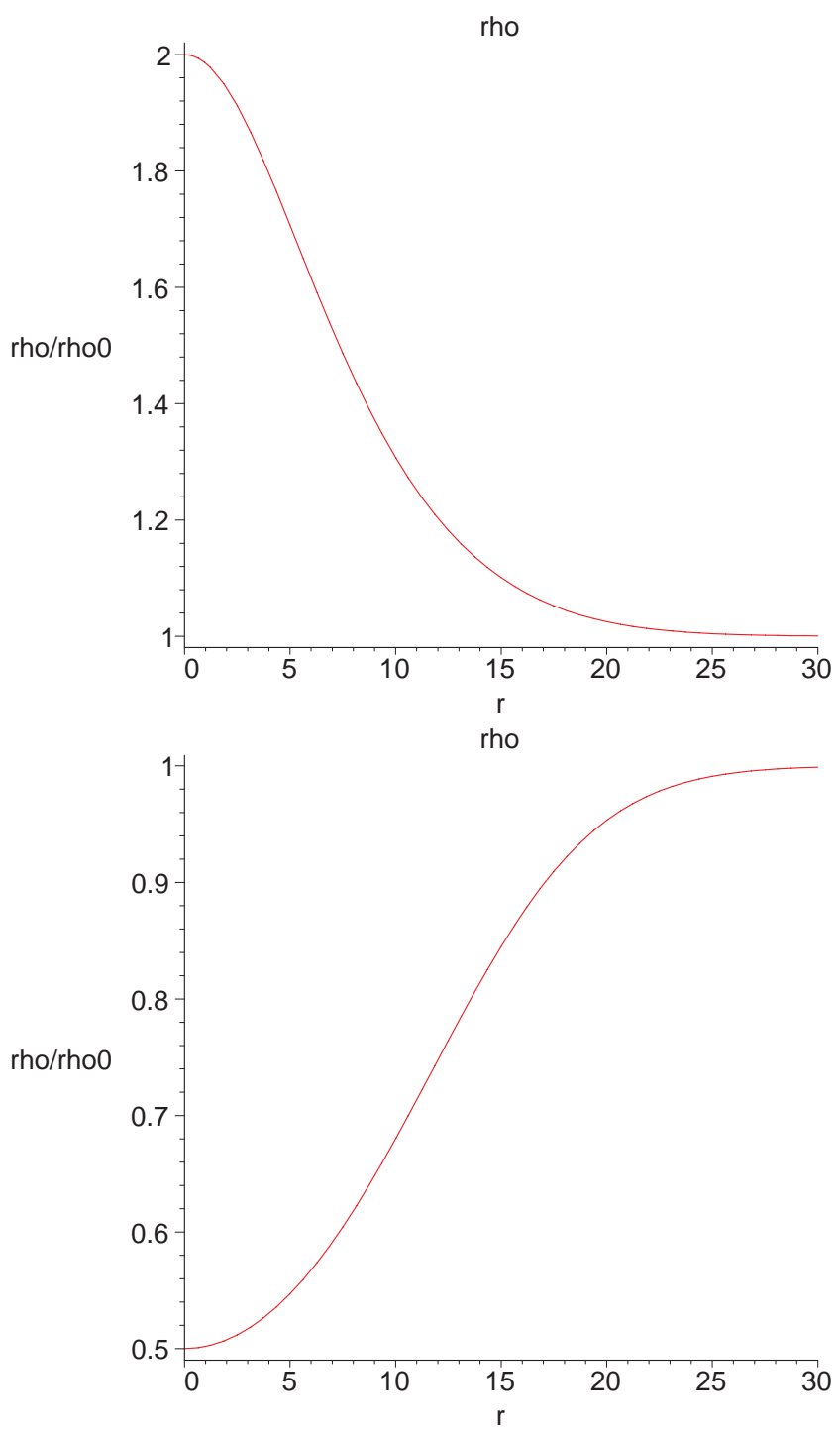

Fig. (1). Plot of the $\rho(r)$ function given in equation (34) for $r_{0}<1$ (top plot) and for $r_{0}>1$ (down plot). The plot is done in terms of $\rho(r) / \rho_{0}$. We have set $r_{0}=2$ and $r_{0}=1 / 2$ for each plot, respectively and $\rho_{0}=0.002$.

For numerical convenience we set $\rho(0) / \rho_{0} \rightarrow r_{0}$ in the plot. This means that if the central density of the BEC is bigger than the density of the ground state, we have the upper profile in Fig. (1), but if it is less than it, we have the bottom profile.

The second and for our interest more interesting limit of equation (33) is when $P>>$. This occurs when the scalar field mass is enough small $m_{\Phi} \ll m_{\text {Planck }}$, as for astrophysical BEC's. In this limit the Oppenheimer-Volkov equation has also an analytical solution given by

$$
\rho(r)=\frac{\rho(0)-\rho_{0}}{\left(2 \pi G r^{2} \omega\left(\rho(0)-\rho_{0}\right)^{5 / 3}+1\right)^{3 / 5}}+\rho_{0},
$$




$$
=\left(\frac{p(0) / \omega}{2 \pi G r^{2} p(0)+1}\right)^{3 / 5}+\rho_{0},
$$

or equivalently $P=1 /\left(2 \pi G r^{2}+1 / P(0)\right)$. In this case the pressure dominates the $\mathrm{BEC}$, the pressure acquire a maximum for $P(0)$. Enough far away from the centre of the BEC we can approximate equation (35) with

$$
\rho=\left(\frac{1 / \omega}{2 \pi G r^{2}}\right)^{3 / 5}+\rho_{0},
$$

which implies a space-time metric for the BEC given by

$$
d s^{2}=\frac{d r^{2}}{1-2\left(r_{0} r^{4 / 5}+\frac{4}{3} \pi G \rho_{0} r^{2}\right)}-\exp (2 v) d t^{2}+r^{2} d \Omega^{2},
$$

where $r_{0}=10 / 9\left(4 \pi^{2} / \omega^{3}\right)^{1 / 5}$. Function $v$ determines the circular velocity (the rotation curves) $V_{r o t}$ of test particles around the BEC. Using the geodesic equation of metric (37) one obtains that $V_{r o t}^{2}=r g_{t t, r} /\left(2 g_{t t}\right)=r v^{\prime} \quad$ [21]. Using equations (27) we can integrate the function $v$ and obtain the rotation curves. The plot is shown in Fig. (2), where we see that the form of the rotation curves are analogous as the expected from the observed in galaxies, specially in LSB and dwarf ones [22-24] besides SFDM predicts a core density profile that could have some advantages [25] over the standard model (cuspy profiles). However, the discussion of the central region of the rotation curves continue. This is the main reason why it is not convenient to tray self-gravitating BECs in the Newtonian limit. Remain that the Newton theory can be derived from the Einstein one for slow velocities, weak fields and pressures much smaller than the densities and this last conditions is not fulfilled in selfgravitating BECs.

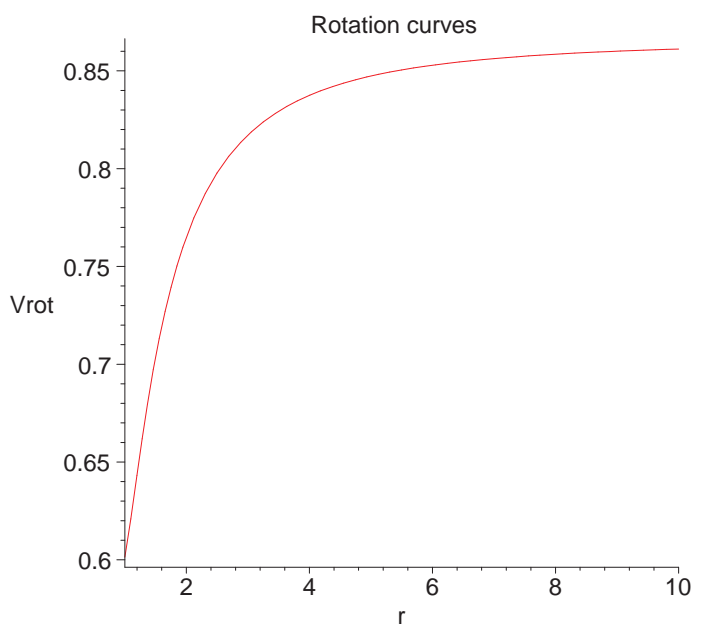

Fig. (2). Rotation curve derived from metric (37). The velocity and the coordinate $r$ are in arbitrary units.

From these results and from the simulations given in [19] it follows a novel paradigm for structure formation, which is different from the bottom-up one. In the SFDM paradigm, after the big bang the scalar field expands till it decouples from the rest of the matter. If the scalar field has a sufficient small mass such that its critical temperature of condensation is less than the temperature of decoupling, the scalar field forms a BEC. Then the scalar field collapses forming objects which final mass is not bigger than the critical mass $m_{\text {Planck }}^{2} / m_{\Phi}$. These objects contain a density profile very similar to the profile shown in the top of Fig. (1). They are very stable under perturbations. It has been proposed that the dark matter in galaxies and clusters is a scalar field with a mass of $10^{-22} \mathrm{eV}$ [15]. If this were the case, the main difference for the structure formation of this ultralight scalar field with the bottom-up paradigm is that the SFDM objects form just after the collapse of the scalar field and remain so during the rest of the Universe expansion. Furthermore, they can collide together but after the collision the objects remain unaltered, since they behave like solitons [26]. This means that in a merging of BEC they pass through each other without some alterations in its total mass as collisionless dark matter. This paradigm implies then that we must be able to see well formed galaxies with the actual masses for very large redshifts, longer than those predicted by the bottom-up paradigm, i.e., by CDM. In this sense some authors [27] suggest a discrepancy between the observed population of massive spheroidal galaxies at high redshift with the numerical simulations of hierarchical merging in a $\Lambda \mathrm{CDM}$ scenario that underpredict this population. However, the discussion continues because other physical processes, as feedback, could have important effects in this galaxies.

\section{THE COSMOLOGY}

In this section we review the Cosmology given by a SFDM model with two different scalar field potentials: $V(\Phi)=\frac{1}{2} m_{\Phi}{ }^{2} \Phi^{2}$ and $V(\Phi)=V_{0}[\cosh (\kappa \lambda \Phi)-1]$ where $m$ is the mass of the boson particle and $V_{0}$ and $\lambda$, free parameters fixed with cosmological data and $\kappa^{2}=8 \pi G$. Based on the current observations from the 5-year WMAP data [28] we will consider a Universe evolving in a spatiallyflat Friedmann Lemaître-Robertson-Walker spacetime. We assume that this Universe contains a real scalar field $(\Phi)$ as dark matter, radiation (r), neutrinos $(v)$, baryons (b) and a cosmological constant $(\Lambda)$ as dark energy.

The total energy density of a homogeneous scalar field is given by

$$
\rho_{\Phi}=\frac{1}{2} \dot{\Phi}^{2}+V(\Phi)
$$

and the radiation and baryonic components are represented by perfect fluids with baryotropic equation of state $p_{\gamma}=(\gamma-1) \rho_{\gamma}$, where $\gamma$ is a constant, $0 \leq \gamma \leq 2$. For example, for radiation and neutrinos $\left(\gamma_{r, v}=\frac{4}{3}\right)$, for baryons $\left(\gamma_{b}=1\right)$ and finally for a cosmological constant $\left(\gamma_{\Lambda}=0\right)$.

Thus, the field equations for a Universe with these components are given by 


$$
\begin{aligned}
& \dot{H}=-\frac{\kappa^{2}}{2}\left(\dot{\Phi}^{2}+\gamma \rho_{\gamma}\right), \\
& \ddot{\Phi}+3 H \dot{\Phi}+\partial_{\Phi} V=0, \\
& \dot{\rho}_{\gamma}+3 \gamma H \rho_{\gamma}=0,
\end{aligned}
$$

and the Friedmann equation

$$
H^{2}=\frac{\kappa^{2}}{3}\left(\rho_{\gamma}+\frac{1}{2} \dot{\Phi}^{2}+V(\Phi)\right) .
$$

In order to analyze the behavior of the different components of this Universe, we are going to use the dynamical system formalism following Appendix 9.

\subsection{The $\Phi^{2}$ Scalar Potential}

We start our cosmological analysis of SFDM taking the potential

$$
V(\Phi)=\frac{1}{2} m^{2} \Phi^{2},
$$

and developing the standard procedure to transform it into a dynamical system. For doing so, the new variables (111) for the system of equations (38) read

$$
\begin{gathered}
x \equiv \frac{\kappa}{\sqrt{6}} \frac{\dot{\Phi}}{H}, u \equiv \frac{\kappa}{\sqrt{6}} \frac{m \Phi}{H}, \\
z_{\gamma} \equiv \frac{\kappa}{\sqrt{3}} \frac{\sqrt{\rho_{\gamma}}}{H} .
\end{gathered}
$$

Using the definitions given in (41), the evolution equations (38) for potential (40) transform into an autonomous system

$$
\begin{aligned}
& x^{\prime}=-3 x-\frac{m}{H} u+\frac{3}{2} \Pi x, \\
& u^{\prime}=\frac{m}{H} x+\frac{3}{2} \Pi u, \\
& z_{\gamma}^{\prime}=\frac{3}{2}(\Pi-\gamma) z_{\gamma}, \\
& -\frac{\dot{H}}{H^{2}}=\frac{3}{2}\left(2 x^{2}+\gamma z_{\gamma}^{2}\right) \equiv \frac{3}{2} \Pi,
\end{aligned}
$$

where as in Appendix 9, prime denotes a derivative with respect to the e-folding number $N=\ln (a)$. Again the choice of phase-space variables (41) transforms the Friedmann equation into a constraint equation

$$
F \equiv x^{2}+u^{2}+z_{\gamma}^{2}=1 .
$$

Because we are considering an expanding Universe which implies that $H>0$ and from the variable definitions (41), we can see that $u, z_{\gamma} \geq 0$. With these variables, the density parameters can be written as

$$
\begin{aligned}
& \Omega_{D M}=x^{2}+u^{2}, \\
& \Omega_{\gamma}=z_{\gamma}^{2},
\end{aligned}
$$

$$
\Omega_{\Lambda}=l^{2},
$$

where we have added explicitly a cosmological constant variable $l \equiv z_{\Lambda}$. Moreover, with the physical constraint $0 \leq \Omega \leq 1$ and the Friedmann equation $\Omega_{D M}+\Omega_{\gamma}+\Omega_{\Lambda}=1$ the variable space is bounded by

$$
0 \leq x^{2}+u^{2}+z_{\gamma}^{2}+l^{2} \leq 1 .
$$

On the other hand, observe that the variable space (42) is not a completely autonomous one because $H$ is an external parameter. In order to close the system we define a new variable $S$ given by

$$
s \equiv \frac{m}{H},
$$

which dynamical equation (112) is

$$
s^{\prime}=\frac{3}{2} \Pi s .
$$

With this new variable, system (42) is now an autonomous one. The whole close system is

$$
\begin{aligned}
x^{\prime} & =-3 x-s u+\frac{3}{2} \Pi x, \\
u^{\prime} & =s x+\frac{3}{2} \Pi u, \\
z_{\gamma}^{\prime} & =\frac{3}{2}(\Pi-\gamma) z_{\gamma}, \\
l^{\prime} & =\frac{3}{2} \Pi l, \\
s^{\prime} & =\frac{3}{2} \Pi s .
\end{aligned}
$$

In order to acquire geometrical information that dynamical system analysis provide (see Appendix 9), we study the stability of (4.1). To do this, we define the vector $\vec{x}=\left(x, u, z_{y}, l, s\right)$ and consider a linear perturbation of the form $\vec{x} \rightarrow \vec{x}+\delta \vec{x}$. The linearized system reduces to $\delta \vec{x}^{\prime}=\mathrm{M} \delta \vec{x}$, where $\mathrm{M}$ is the Jacobian matrix of $\vec{x}^{\prime}$ and it reads as

$$
M=\left(\begin{array}{ccccc}
\frac{3}{2} \Pi-3+6 x^{2} & -s & 3 \gamma x z & 0 & -u \\
6 x u+s & \frac{3}{2} \Pi & 3 \gamma u z & 0 & x \\
6 x z & 0 & \frac{3}{2} \Pi+3 \gamma z^{2}-\gamma & 0 & 0 \\
6 x l & 0 & 3 \gamma l z & \frac{3}{2} \Pi & 0 \\
6 x s & 0 & 3 \gamma s z & 0 & \frac{3}{2} \Pi
\end{array}\right) .
$$


The equilibrium points $\vec{x}_{c}$ of the phase space $\left\{x, u, z_{\gamma}, l, s\right\}$, considering only $\gamma=4 / 3$, are then

1. $\{ \pm 1,0,0,0,0\}$ Kinetic scalar domination

2. $\{0,0,1,0,0\}$ Radiation domination

3. $\{0,0,0,1, s\}$ Cosmological constant domination

4. $\{0, u, 0, l, 0\}$ Cosmological constant and Potential scalar domination

And finally, the eigenvalues of the matrix $\mathcal{M}$ valued at the critical points listed above read

1. $\{6,3,3,3,3-\gamma\}$

2. $\left\{\frac{3 \gamma}{2}, \frac{3 \gamma}{2}, \frac{3 \gamma}{2}, \frac{7 \gamma}{2}, \frac{3}{2}(-2+\gamma)\right\}$

3. $\left\{0,0, \frac{1}{2}\left(-3-\sqrt{9-4 s^{2}}\right), \frac{1}{2}\left(-3+\sqrt{9-4 s^{2}}\right),-\gamma\right\}$

4. $\{-3,0,0,0,-\gamma\}$

As we can see, the radiation domination epoch shows a saddle point, however, in order to reproduce the big bang nucleosynthesis process is necessary that this kind of matter would had dominated in the past of the Universe. In other words, the radiation points should have corresponded to a source point. The domination of dark matter in the past (a source point) and the cosmological constant in the future (an attractor point) are showed in the Fig. (3).

In the following, we integrate system (46) with the constraint (43), following the procedure shown in Appendix 9. In general this system is very difficult to integrate because it is a non-linear four-dimensional differential system of equations. It is clear that system (46) is a complete system which can fulfil or not the constraint (43). However, as it was shown in Appendix 9 system (46) together with constraint (43) is completely integrable. For simplicity we will take all the perfect fluid components as the equation $z_{\gamma}{ }^{\prime}=3 / 2(\Pi-\gamma) z_{\gamma} \quad$ with the Friedman equation $x^{2}+u^{2}+z_{\gamma}^{2}=1$.

Thus, we substitute $3 / 2 \Pi$ from equation (46e) into the rest of the equations. With this substitution equation (46e) integrates in terms of $s$ as

$$
z_{\gamma}=\sqrt{\Omega_{0 \gamma}} \operatorname{sexp}\left(-\frac{3}{2} \gamma N\right)
$$

where $\Omega_{0 \gamma}$ is an integration constant. We multiply (46a) by $2 x$ and (46b) by $2 u$ and sum both equations. We obtain

$$
\left(x^{2}+u^{2}\right)^{\prime}=-6 x^{2}+2 \ln (s)^{\prime}\left(x^{2}+u^{2}\right) .
$$

Now, we use constraint (43) and equation (47) into equation (48) to obtain

$$
6 x^{2}=2 \ln (s)^{\prime}-3 \gamma s^{2} \Omega_{0 \gamma} \exp (-3 \gamma N) .
$$

We substitute (49) and (47) into (46e) to obtain $0=0$. Therefore, $s$ is not an independent variable and we cast it into the system as a control parameter, the same result is found by [29]. In what follows we will use this important result.

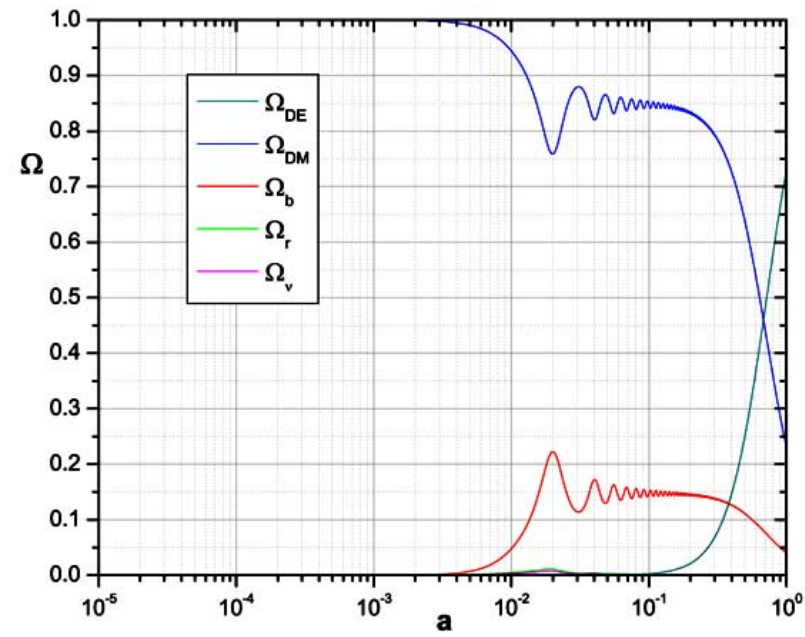

Fig. (3). Evolution of the density parameters for the system of equations (4.1). The plot shows the dark matter domination epoch at early times, a source point. The cosmological constant at the future of the Universe is an attractor point.

Of course, to guess variable $s$ in order to fulfil constrain (43) is not so easy. In order to avoid this problem we can consider the observed dynamic for $H$ and model it by the following ansatz

$$
H \equiv \frac{t_{0}{ }^{n-1}}{t^{n}},
$$

because it is well-know the behavior for $H$ at different epochs

$$
H_{\text {dust }}=\frac{2}{3 t}, H_{r a d}=\frac{1}{2 t}, H_{\Lambda}=\sqrt{\frac{\Lambda}{3}} .
$$

There exists a restriction in the parameter $n$. Because is well know that $H$ is a function monotonically decreasing, $n$ has to satisfy $n \geq 0$.

With the ansatz (50), the dynamical equation for $s$ reads

$$
s^{\prime}=\left(m t_{0}\right)^{\frac{1}{n}-1} n\left(\frac{1}{s}\right)^{\frac{1}{n}-2}=s_{0} s^{-k},
$$

where we have defined $k \equiv 1 / n-2$.

In the following, we investigate if this system can reproduce the observed Universe. We introduce the components of the background Universe into the dynamical system described by (4.1) adding to it baryons $(b)$, radiation $(z)$ and neutrinos $(\boldsymbol{v})$. Thus, the system transforms into 


$$
\begin{array}{r}
x^{\prime}=-3 x-s u+\frac{3}{2} \Pi x, \\
u^{\prime}=s x+\frac{3}{2} \Pi u, \\
b^{\prime}=\frac{3}{2}(\Pi-1) b, \\
z^{\prime}=\frac{3}{2}\left(\Pi-\frac{4}{3}\right) z, \\
v^{\prime}=\frac{3}{2}\left(\Pi-\frac{4}{3}\right) v, \\
l^{\prime}=\frac{3}{2} \Pi l, \\
s^{\prime}=s_{0} s^{-k},
\end{array}
$$

with $\Pi=2 x^{2}+b^{2}+\frac{4}{3} z^{2}+\frac{4}{3} v^{2}$ and the Friedmann equation reduces to the constraint

$$
F=x^{2}+u^{2}+b^{2}+z^{2}+v^{2}+l^{2}=1 .
$$

Using this ansatz we can reduce till quadratures the solution of system (3). In order to do this, observe that

$$
\frac{3}{2} \Pi=s_{0} s^{-k-1} .
$$

Using this last identity, equation (53c)-(54f) can be integrated to give

$$
z_{\gamma}=z_{0}\left[s_{0}(k+1) N+s_{1}\right]^{\frac{1}{k+1}} e^{-\frac{3}{2} \gamma N},
$$

for each corresponding value of $\gamma$. Finally, equations (53a) and (53b) can be integrated as follows. We divide (53a) by $x$ and (53b) by $u$ and take the difference between both equations. We define $y=x / u$ to obtain

$$
y^{\prime}+3 y+q(N) y^{2}=-q(N),
$$

where function $q(N)=\left[s_{0}(k+1) N+s_{1}\right]^{1 /(k+1)}$. Equation (55) is a Riccati equation which can be reduce to a Bernoulli equation by defining $y=w+y_{1}$, where $y_{1}$ is a known solution of (55). It reduces to

$$
w^{\prime}+\left(3+2 q y_{1}\right) w+q z^{2}=0 .
$$

Equation (56) can be further reduced by defining $W=1 / w$, we obtain

$$
W^{\prime}-\left(3+2 q y_{1}\right) W-q=0,
$$

which integral is

with $A=\int\left(3+2 q y_{1}\right) d N$.

Thus

$$
\begin{array}{r}
u=u_{0} q \exp \left(\int y q d N\right), \\
x=x_{0} q e^{-3 N} \exp \left(-\int \frac{q}{y} d N\right), \\
z_{\gamma}=z_{0} q e^{-\frac{3}{2} \gamma N}, \\
y=\frac{1}{W}+y_{1} .
\end{array}
$$

In the particular case where $s_{0}=0$, the integrals can be solved analytically, however this value for $s_{0}$ does not have a physical meaning.

On the other hand, we can evaluate the integrals using numerical methods for different values of the free constants. We can obtain a numerical solution for the system using (59)
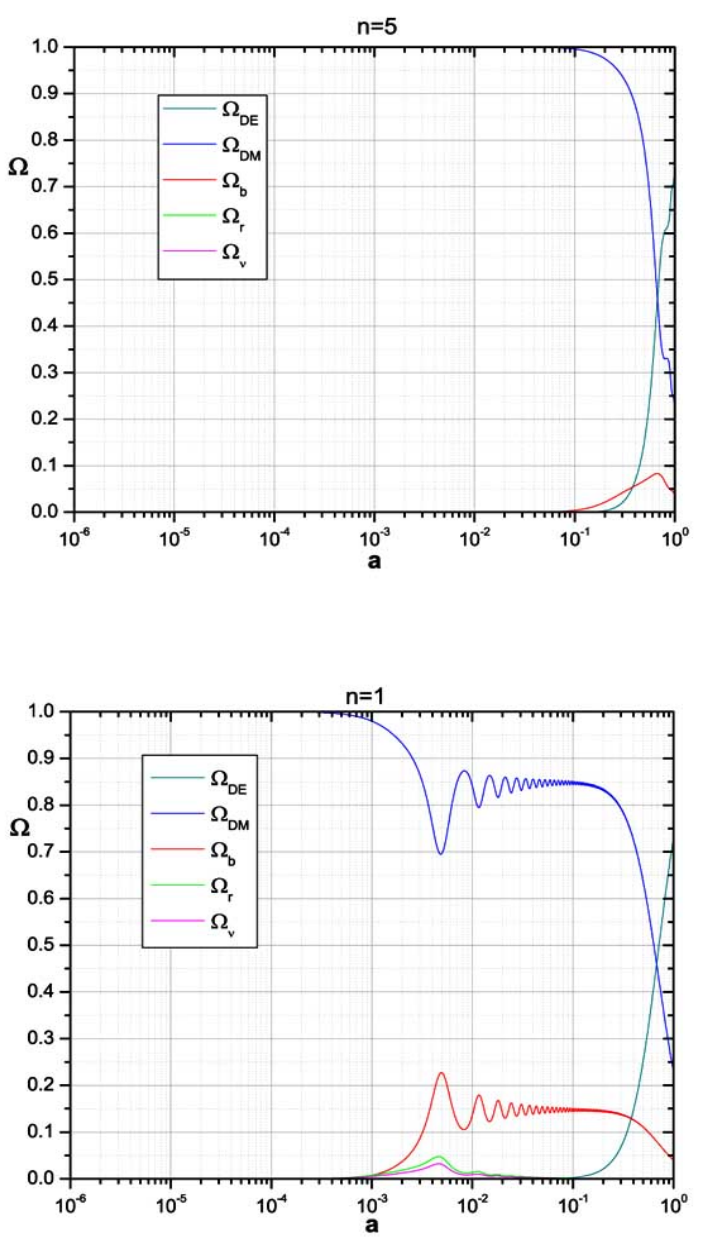

Fig. (4). Evolution of the density parameters for the system (3) with $n=1$ (top panel) and $n=5$ (bottom panel). This values of $\mathrm{n}$ are not reproduce the standard behavior of $\Lambda \mathrm{CDM}$. 

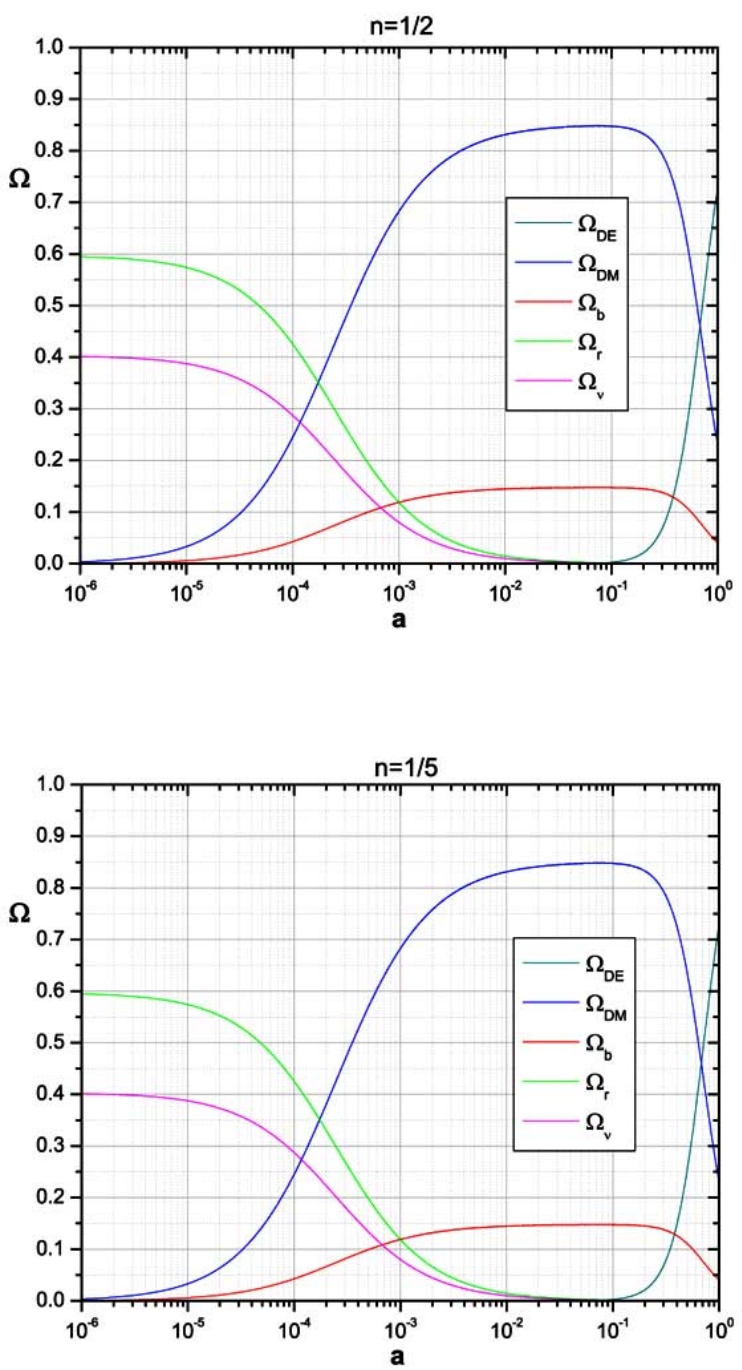

Fig. (5). Upper panel: evolution of the density parameters for the system (3) with $n=1 / 2$. Lower panel: evolution of the density parameters for the system 3 with $n=1 / 5$. SFDM reproduces the standard $\Lambda \mathrm{CDM}$ behavior in both cases.

or directly integrating system (53) with an AdamsBashforth-Moulton (ABM) method and using as initial data the WMAP+BAO+SN recommended values to $\Omega_{\Lambda}^{(0)}=0.721$, $\Omega_{D M}^{(0)}=0.233, \quad \Omega_{b}^{(0)}=0.0454, \quad \Omega_{r}^{(0)}=0.0004$, $\Omega_{v}^{(0)}=0.0002$, the result is the same.

Fig. (4) and Fig. (5) show the numerical solutions of the dynamical system (53). In Fig. (4) we set $n \geq 1$, as examples we show $n=1,5$. From these figures it is clear that the radiation remains subdominant for this values of $n$. Fig (6) shows the constraint $F$ in (54) in order to visualize the integration's error. Observe that $F=1$ at every point in the evolution, indicating that the Friedmann equation is exactly fulfilled all the time, this behavior is exactly the same for all runs. On the other hand, in Fig. (5), where the plots were made for $n=1 / 2,1 / 5$, the radiation and the neutrinos behave exactly in the same way as they do in the $\Lambda \mathrm{CDM}$ model so

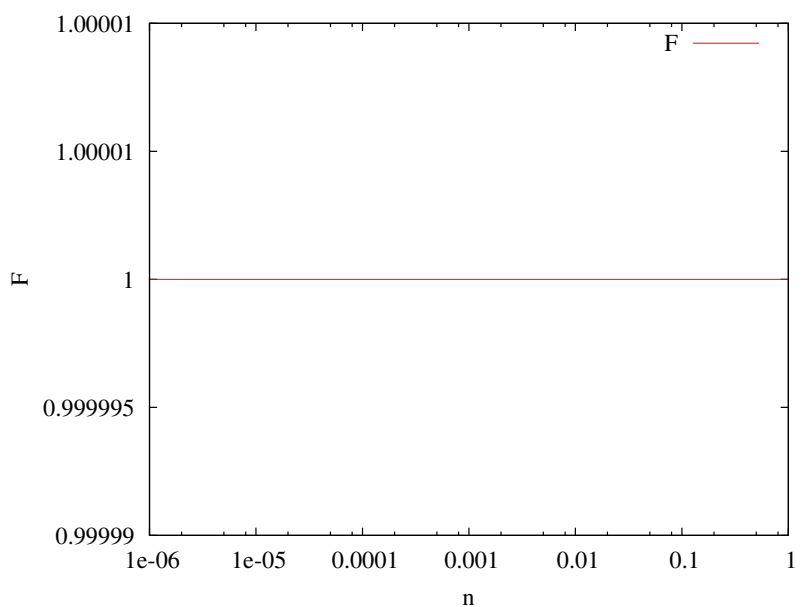

Fig. (6). Evolution of the function $F=x^{2}+u^{2}+b^{2}+z^{2}+v^{2}+l^{2}$ in (54) for the system (3) with $n=1,5,1 / 2$ and $1 / 5$. Function $F$ is exactly the same for all values of $n$ in all these cases.

we expect that both of these can reproduce the observed Universe. The first values for $n$ are not able to explain the big bang nucleosynthesis, since radiation never dominates as it is required. However, the last values for $n$ can reproduce the radiation dominated era. Following the radiation dominated era, $\Phi^{2}$ dark matter become the component that dominates the evolution and finally the Universe is dominated by the cosmological constant.

\subsection{The Cosh Scalar Potential}

Now, we are going to compare above results with the potential

$$
V(\Phi)=V_{0}[\cosh (\kappa \lambda \Phi)-1]
$$

In order to do so, we define new variables as

$$
\begin{aligned}
& x=\frac{\kappa}{\sqrt{6}} \frac{\dot{\Phi}}{H}, \\
& u=\sqrt{\frac{2 V_{0}}{3}} \frac{\kappa}{H} \cosh \left(\frac{1}{2} \kappa \lambda \Phi\right), \\
& v=\sqrt{\frac{2 V_{0}}{3}} \frac{\kappa}{H} \sinh \left(\frac{1}{2} \kappa \lambda \Phi\right), \\
& z_{\gamma}=\frac{\kappa}{\sqrt{3}} \frac{\sqrt{\rho_{\gamma}}}{H}, l=\frac{\kappa}{\sqrt{3}} \frac{\sqrt{\rho_{\Lambda}}}{H} .
\end{aligned}
$$
obtain

Substituting definitions (61) into equations (61) we

$$
\begin{aligned}
& x^{\prime}=-3 x-\lambda v u+\frac{3}{2} \Pi x, \\
& u^{\prime}=\lambda x v+\frac{3}{2} \Pi u,
\end{aligned}
$$




$$
\begin{aligned}
& v^{\prime}=\lambda x u+\frac{3}{2} \Pi v, \\
& z_{\gamma}{ }^{\prime}=\frac{3}{2}(\Pi-\gamma) z_{\gamma}, \\
& l^{\prime}=\frac{3}{2} \Pi l,
\end{aligned}
$$

where again the prime means derivatives with respect the efolding number $N=\ln (a)$ and we are also using the function $\Pi=2 x^{2}+\gamma z^{2}$. From the definitions (61) it follows the constraints

$$
u^{2}-v^{2}=\frac{2 V_{0} \kappa^{2}}{3} \frac{1}{H^{2}}=\frac{1}{\lambda^{2}} \frac{m_{\Phi}^{2}}{H^{2}},
$$

and the Friedmann equation (43) written in this variables reads

$$
F=x^{2}+u^{2}+z^{2}+l^{2}=1 .
$$

However, equation (64) is actually not real constraint, since they are inhered in the dynamical equations (62) (see appendix A equation (115). Furthermore, constraint (63) is also inhered in the dynamical system, observe that if we multiply the second equation of (62) by $1 / 2 u$ and the third by $1 / 2 v$ and rest each other, we obtain

$$
H^{\prime}=-\frac{3}{2} \Pi H
$$

But this relation follows directly from the field equations (38). This means that system (62) is compatible with the constraint (63). Using this constraint (63) in the dynamical system (62), we obtain

$$
\begin{aligned}
& x^{\prime}=-3 x-u \sqrt{\lambda^{2} u^{2}+\left(\frac{m}{H}\right)^{2}}+\frac{3}{2} \Pi x, \\
& u^{\prime}=x \sqrt{\lambda^{2} u^{2}+\left(\frac{m}{H}\right)^{2}}+\frac{3}{2} \Pi u, \\
& z^{\prime}=\frac{3}{2}(\Pi-\gamma) z, \\
& l^{\prime}=\frac{3}{2} \Pi l .
\end{aligned}
$$

we notice, that occurs the same situation as $\Phi^{2}$ potential. Introducing again the variable $s=m / H$ with its dynamical equation.

$$
s^{\prime}=\left(m t_{0}\right)^{\frac{1}{n}-1} n\left(\frac{1}{s}\right)^{\frac{1}{n}-2},
$$

$$
\begin{aligned}
& x^{\prime}=-3 x-u \sqrt{\lambda^{2} u^{2}+s^{2}}+\frac{3}{2} \Pi x, \\
& u^{\prime}=x \sqrt{\lambda^{2} u^{2}+s^{2}}+\frac{3}{2} \Pi u, \\
& z_{\gamma}^{\prime}=\frac{3}{2}(\Pi-\gamma) z_{\gamma}, \\
& l^{\prime}=\frac{3}{2} \Pi l, \\
& s^{\prime}=s_{0}\left(\frac{1}{s}\right)^{\frac{1}{n}-2} .
\end{aligned}
$$

The density parameters are the same as we have defined at (104). We solve numerically (68) with the same initial conditions like the system of equations (53) and $\lambda \approx 20$. The plots are shown in Fig. (7).

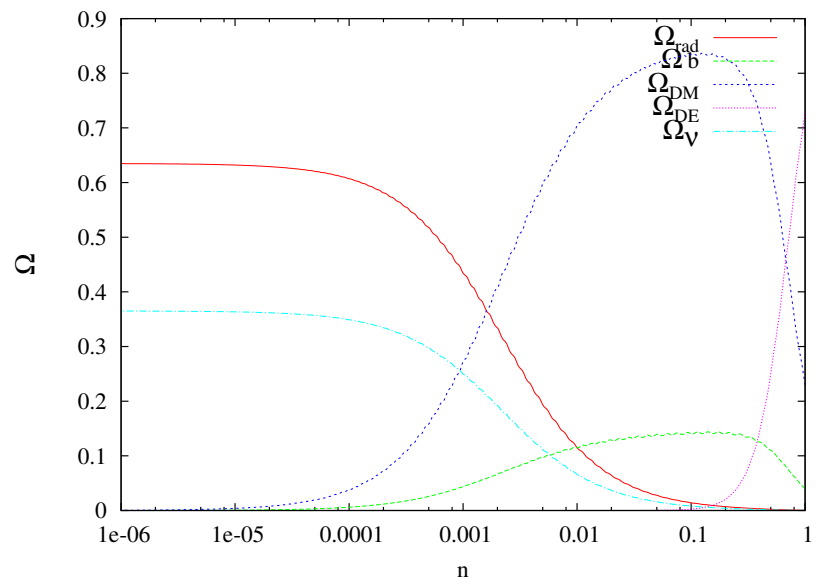

Fig. (7). Evolution of the density parameters for the system (68), where the scalar field potential is given by the equation (60).

The plot shows the dynamical evolution for a Universe with SFDM with the potential (53), notice that is equivalent to potential (60).

Finally, we use the same dynamical system formalism for the case of $\Lambda \mathrm{CDM}$ in order to compare with SFDM. We consider that it background Universe is composed by baryons, radiation, neutrinos, dark matter and cosmological constant with an equation of state as perfect fluid. We solve numerically this system and in general terms the dynamic of both scalar potential is indistinguishable of the standard model. This is an important goal of this paper.

The next step is to compute the age of the Universe using our model. The age equation can be written as

$$
t_{o}=\int_{N_{o}}^{N} \frac{1}{H} d N
$$

we obtain 
using the definition for $l$ from (41) or (61) eq. (61) reduces to

$$
t_{o}=\frac{\sqrt{3}}{\kappa \sqrt{\rho_{\Lambda}}} \int_{N_{o}}^{N} l d N
$$

we compute (70) and obtain that $t_{o} ; 13.77$ Gyr. This result is in agreement with the cosmological observations from $\mathrm{WMAP}+\mathrm{BAO}+\mathrm{SN}$ which estimate $t_{o}=13.73 \pm 0.12 \mathrm{Gyr}$ and therefore $H_{o}=70.1 \pm 1.3 \mathrm{~km} / \mathrm{s} / \mathrm{Mpc}$. Furthermore, in Fig. (7), we see that scale factor of decoupling is $a: 10^{-3}$, this means a redshift $z: 1000$. At this redshift, the neutrinos made up : $12 \%$ of the Universe. On the other hand, WMAP cosmological observations show that when the Universe was only 380,000 years old, neutrinos permeate the Universe within $10 \%$ of its total energy density. Thus, SFDM is in concordance within the measurements of WMAP. This result shows that scalar field is a plausible candidate for dark matter because it behave like cold dark matter.

\section{QUANTUM EFFECTS OF THE SCALAR FIELD}

SFDM particles have a mass of $m_{S F D M}: 10^{-22} \mathrm{eV}$, with this mass the quantum mechanics effects are important at the scale of $: 1 / m_{S F D M}: 1 \mathrm{pc}$. In this section we show that if the SFDM are the dark matter, quantum effects of the ultralight particles can explain a constant density on the background of the universe and can be interpreted as a cosmological constant.

In order to do so, we write the Schrödinger equation in its hydrodynamical version,

$$
\begin{aligned}
& \frac{\partial \rho}{\partial t}+\nabla \cdot(\rho v)=0 \\
& \frac{\partial(\rho v)}{\partial t}+\nabla \cdot(\rho v v)=F_{Q}-\frac{\rho}{m} \nabla U
\end{aligned}
$$

Equations (71) are equivalent to the Schrödinger equation if the wave function $\psi$ is decomposed as $\psi=\sqrt{\rho} \exp (i S)$, being $v=\hbar / m \nabla S$. The main difference of equations (71) and the hydrodynamical equations is the quantum force $F_{Q}=-\nabla U_{Q}$, which is derivable from the quantum potential

$$
U_{Q}=-\frac{\hbar^{2}}{2 m^{2}} \frac{1}{\sqrt{\rho}}\left(\nabla^{2} \sqrt{\rho}\right)
$$

$U_{Q}$ is interpreted as the quantum mechanical contribution to the hydrodynamical equations. If we drop out the quantum force $F_{Q}$ in equation (71), these equations are the classical equations for a hydrodynamical system. Of course, the universe is quantum mechanical but the contribution of $F_{Q}$ to the hydrodynamical equations is very small due to the factor $\frac{\hbar^{2}}{2 m^{2}}$, which make this force very small for a mass $m$ of a normal classical object. But for an SFDM field of mass $m_{S F D M}: 10^{-22} \mathrm{eV}$ this term can not be neglected anymore.

Of course the SFDM field, if it is the dark matter in the universe, is not longer uniformly distributed in the universe. The dark matter forms clusters of galaxies, galaxies, etc. We can estimate the density or the square root of the density in the following way.

We analyse the behaviour of the quantum density $\rho$ in a short time variation, in a region of the universe where the space-time is almost flat, if we decompose the scalar field as $\Phi=\psi \exp (-i m t)+c . c$. , the Klein-Gordon equation reduces to

$$
\frac{\hbar^{2}}{2 m^{2} c^{2}} \ddot{\psi}+i \hbar \dot{\psi}+\frac{\hbar^{2}}{2 m} \nabla^{2} \psi=0
$$

In terms of the decomposition $\psi=\sqrt{\rho} \exp (i S)$, we can further decompose $\sqrt{\rho}=\sqrt{\rho_{k^{\prime}}} \exp \left(i k^{\prime} t\right)$, and neglect for the moment the variation of $S$, we arrive at

$$
\nabla^{2} \sqrt{\rho_{k^{\prime}}}-\sqrt{\rho_{k^{\prime}}}\left(k^{\prime 2}+\frac{2 k^{\prime} m}{\hbar}\right)=0
$$

On the other hand, the distribution of the dark matter can be modeled as consentrations of matter, for example as Dirac delta functions distributions $\sqrt{\rho}: \delta\left(r-r_{0}\right)$. But, if we accept the cosmological principle, the dark matter clumps are homogeneously distributed in the universe, one can suppose that if there exists a galaxies in $r_{0}$, it should exist another galaxy in more of less $-r_{0}$, thus we can put these ideas together if we write that $\sqrt{\rho}: \delta\left(r-r_{0}\right)+\delta\left(r+r_{0}\right)=2\left|r_{0}\right| \delta\left(r^{2}-r_{0}^{2}\right)$. It is well known that the delta function can be written as $\delta\left(a r^{2}-1\right)=\lim _{l \rightarrow \infty} l J_{l}\left(a l r^{2}\right)$, where $J_{l}$ are the Bessel functions. In other words, it is convenient to expand the density distribution of the galaxies as

$$
\sqrt{\rho}=\sum_{l m} U_{l m} \frac{J_{l+\frac{1}{2}}\left(k r^{2}\right)}{\sqrt{r}} Y_{m}^{l}(\theta, \varphi)
$$

where $k$ is the wave number of the minimal scale of fluctuations of the dark matter with units of $L^{-2}$ and $Y_{m}^{l}$ are the spherical harmonic functions with $m=2 l+\frac{1}{2}$. Of course, any function can be expanded as equation (75), because the Bessel and the spherical harmonic functions are basis of the 
functions space. Now we are able to calculate the quantum potential $U_{Q}$, we obtain

$$
U_{Q}=-\frac{\hbar^{2}}{2 m^{2}} \frac{1}{\sqrt{\rho}}\left(\nabla^{2} \sqrt{\rho}\right)=\frac{\hbar^{2}}{2 m^{2}} k^{2} r^{2}
$$

$U_{Q}$ is the potential that effectively feel the galaxies in a cluster due to the quantum effects of the SFDM. The effective density can be obtained with the Poisson equation $\nabla^{2} U_{Q}=4 \pi G \rho_{\text {eff }}$ using (76) as a source of the density. We get

$$
\rho_{\text {eff }}=3 \frac{k^{2} \hbar^{2}}{\pi G} \frac{1}{m_{D M}^{2}}
$$

The first result we observe here is that the effective density is constant, it does not depend on the position. This is a remarkable result, because we see that quantum effects cannot be neglected for masses of the order of the SFDM mass and its effective density is constant. Nevertheless, this effective density does not have to be of the size of something well know.

We set the observed values of the different quantities in this formulae. The smallest structure we observe in the galaxies are the dwarf and satelites galaxies, with a size of $10 \quad \mathrm{kpc}, \quad$ thus $\quad k \sim\left(\frac{2 \pi}{10}\right)^{2} \frac{1}{\mathrm{kpc}^{2}} \sim\left(6.4 \cdot 10^{-37} \mathrm{GeV}\right)^{2}$; $m_{S F D M} \sim 10^{-22} \mathrm{eV}$, we obtain that

$$
\rho_{\text {eff }} \sim\left(6.4 \cdot 10^{-37} \mathrm{GeV}\right)^{4} \frac{m_{P l}^{2}}{m_{D M}^{2}} \sim 4 \cdot 10^{-47} \mathrm{GeV}^{4}
$$

This is a real surprise, the effective density due to the quantum effects of the a particle like the SFDM is of the order of the critical density of the universe $\rho_{\text {crit. }}=8.099 h^{2} 10^{-47} \mathrm{GeV}^{4}$, that means, it is possible that the constant energy we feel in the universe is due quantum effects of small particles of dark matter.

In this section we have shown that, using very simple calculations, that a particle of a small mass has quantum effects at classical scales. This is not a surprise, because we know that the quantum effects of a particle are of the order of the de Broglie length, inverse to the mass of the particle $: 1 / \mathrm{m}$. The first remarkable result is that with very simple arguments but using the hydrodynamical version of the Schrödinger equation, we can see that the effective density of the quantum effects are constant. The great surprise is that the amount of this density is just of the order of magnitude of the critical density of the universe, which coincide almost with the amount of dark energy in the universe.

Thus, there is no contribution of the cosmological constant as long as the quantum density $\rho$ is homogeneous. But, it is important when the structure of the universe begins to form. This is a very elegant way to solve the coincidence problem.

Of course, the fact that we do not see the supersymmetric particles in the detectors is not an argument to discard them as dark matter. The SFDM is also a very good candidate to be the dark matter, fortunately without the problems of the WIMPs, like the cuspy central densities in galaxies, the excess of substructure, etc. (see for example [30, 31]. However, if the SFDM remains as candidate of the dark matter of the universe, we have to take into account the quantum effects of this particle in the background, they cannot be neglected.

\section{PERTURBATION THEORY}

In this section we compute the growth of the overdensities in the regime when the density contrast $\delta=1$. Now a days it is known that our Universe in not exactly isotropic and spatially homogeneous like the FLRW equations describe. There exist small deviations from this model, and if we believe this deviations are small enough, they can be treated by Linear Perturbation Theory.

We consider a flat, homogeneous and isotropic background Universe,were we use the Friedmann-LemaitreRobertson-Walker (FLRW) metric with scale factor $a(t)$ and our background Universe is composed by SFDM ( $\Phi)$. The equations that describe this Universe are (we set $c=\hbar=1)$.

We begin by recalling the basic background equations. From the energy-momentum tensor we have the energy density and the pressure;

$$
\begin{aligned}
& T_{0}^{0}=-\rho=-\left(\frac{1}{2} \dot{\Phi}_{0}^{2}+V_{0}\right) \\
& T_{j}^{i}=p=\left(\frac{1}{2} \dot{\Phi}_{0}^{2}-V_{0}\right) \delta_{j}^{i}
\end{aligned}
$$

Einstein's equations give us the Friedmann equations.

$$
\begin{aligned}
& 8 \pi G \rho=3 H^{2} \\
& 4 \pi G(\rho+3 p)=-3 \dot{H}
\end{aligned}
$$

So, from the above equations we can notice than when talking about a FLRW background, the only quantities at zero order are the energy density $\rho$, pressure $\mathrm{p}$ and volumetric expansion 3H.Finally we have also to remember that $\mathrm{SF}$ are gobernated by Klein-Gordon's equation, which gives the evolution for the SF in a homogeneous expanding Universe,

$$
\ddot{\Phi}_{0}+3 H \dot{\Phi}_{0}+V,_{\Phi}=0
$$

From the theory above we have the basic equations for treating with the non-perturbed space of FLRW.In this 
section we consider perturbations to first order in a FLRW background, so we can separate the metric tensor as follows:

$$
g_{\mu v}=g_{\mu \nu}^{(0)}+\delta g_{\mu v}
$$

From this we get the most general perturbed line element

$$
\begin{aligned}
& d s^{2}=a^{2}(\eta)-\left((1+2 \phi) d \eta^{2}+2 B,{ }_{i} d \eta d x^{i}\right. \\
& \left.+\left[(1-2 \psi) \delta_{i j}+2 E,{ }_{i j}\right] d x^{i} d x^{j}\right)
\end{aligned}
$$

Where $\phi, B, \psi$ and $E$ are the perturbations to the metric and $\delta_{i j}$ is the background metric.

By definition, a perturbation done on any quantity, is the difference between its value in some event in real spacetime, and its corresponding value in the background. We separate the different quantities in a background value and a perturbation

$$
\mathbf{T}=\mathbf{T}_{0}+\delta \mathbf{T}
$$

where the background value is a time dependent quantity only $\mathbf{T}_{0} \equiv \mathbf{T}_{0}(t)$, while the perturbations depend on time and also on the space coordinates $\delta \mathbf{T} \equiv \delta \mathbf{T}\left(x^{i}\right)$ where $x^{\mu}=\left[t, x^{i}\right]$.

With this considerations at hand we have the perturbed Einstein's equations $\delta G_{j}^{i}=8 \pi G \delta T_{j}^{i}$ for a $\mathrm{SF}$ using the Newtonian gauge in which only the scalar perturbations are taken into account. Another advantage in using this gauge is that both potentials $\phi$ and $\psi$ are identical. The Newtonian gauge is then defined when, $B=0$ and $E=0$ Then we have

$$
\psi-\phi=0
$$

where equation (87) usually contains a term of anisotropic stress on the right hand side. In the case of a SF this term vanishes.

In solving the evolution equations it is common to work in Fourier space instead of real space. The beauty of this expansion lying in the fact that each Fourier mode propagates independently.

At first order, the change to Fourier components its done implicitly.A perturbation $\delta \Phi$ is related to its Fourier component $\delta \Phi_{\mathrm{k}}$ like:

$$
\delta \Phi\left(t, x^{i}\right)=\int d^{3} k \delta \Phi\left(t, k^{i}\right) \exp \left(i k_{i} x^{i}\right)=\int d^{3} k \delta \Phi_{\mathbf{k}} \exp \left(i k_{i} x^{i}\right),
$$

where $k^{i}$ is the wave number, and is defined as $k=2 \pi / \lambda$ were $\lambda$ is the length scale of the perturbations.

The equations for the perturbed SF in Fourier space are then:

$$
-4 \pi G \dot{\Phi}_{0} \delta \dot{\Phi}_{\mathbf{k}}=4 \pi G\left(3 H \dot{\Phi}_{0} \delta \Phi_{\mathbf{k}}+V,_{\Phi} \delta \Phi_{\mathbf{k}}-\dot{\Phi}_{0}^{2} \phi\right)+\frac{k^{2}}{a^{2}} \psi
$$

$$
\begin{aligned}
& \dot{\psi}=4 \pi G \dot{\Phi}_{0} \delta \Phi_{\mathbf{k}}-H \phi \\
& H \dot{\phi}=4 \pi G\left(\dot{\Phi}_{0} \delta \dot{\Phi}_{\mathbf{k}}-\dot{\Phi}_{0}^{2} \phi-V,_{\Phi} \delta \Phi_{\mathbf{k}}\right) \\
& -\ddot{\psi}-3 H \dot{\psi}-\left(2 \dot{H}+3 H^{2}\right) \phi \\
& +\frac{k^{2}}{2 a^{2}}(\phi-\psi)+\frac{1}{2 a^{2}}(\phi-\psi)_{\mid j}^{i}
\end{aligned}
$$

With the generalized Klein-Gordon equation as:

$\delta \ddot{\Phi}_{\mathbf{k}}+3 H \delta \dot{\Phi}_{\mathbf{k}}+\left(\frac{k^{2}}{a^{2}}+V,_{\Phi \Phi}\right) \delta \Phi_{\mathbf{k}}=-2 V,_{\Phi} \phi+\dot{\Phi}_{0} \dot{\phi}+3 \dot{\Phi}_{0} \psi$

This equations describe the evolutions of the perturbations, (88a) refers to the energy density, (88b) to the gravitational potential and finally (89) refers to the perturbations in our scalar field.In all the above equations, the perturbed quantities correspond to the perturbations amplitude that are found in the k-th mode.

We now study the qualitative properties of our cosmological model, using a dynamical system. To simplify our system of equations we introduce the next dimensionless variables;

$$
\begin{aligned}
& \frac{\dot{H}}{H^{2}}=-\frac{3}{2} \pi, \\
& \frac{\dot{\Phi}_{0}}{H}=\frac{\sqrt{6}}{\kappa} x, \\
& \frac{V, \Phi_{\Phi}}{H^{2}}=-\frac{\sqrt{6}}{\kappa} U, \\
& x_{1}=\psi, \\
& \frac{\dot{\psi}}{H}=x_{2} \Rightarrow \frac{\ddot{\psi}}{H^{2}}=\frac{\dot{x}_{2}}{H}-\frac{3}{2} \pi x_{2}, \\
& \kappa \delta \Phi_{\mathbf{k}}=\sqrt{6} z_{1}, \\
& \kappa \frac{\delta \dot{\Phi}_{\mathbf{k}}}{H}=\sqrt{6} z_{2} \Rightarrow \frac{\delta \Phi_{\mathbf{k}}}{H^{2}}=\frac{\sqrt{6}}{\kappa} \frac{\dot{z}_{2}}{H}-\frac{\sqrt{6}}{\kappa} \frac{3}{2} \pi z_{2}, \\
& l_{1}=\phi, \\
& \frac{\dot{\phi}}{H}=l_{2}, \\
& \frac{V_{0}}{H^{2}}=3 \frac{u^{2}}{\kappa^{2}} \\
& s=\frac{1}{H},
\end{aligned}
$$

where $\kappa^{\prime 2}=4 \pi G$ and $\pi=2 x^{2}+\gamma z^{2}$. Using these variables (90), we have our autonomous dynamical system with respect to $n$, with $n=\ln a$, or, $\frac{d}{d t}=H \frac{d}{d n}$, and ' denotes the 
derivative with respect to the e-folding $\mathrm{n}$. Taking into account the relation $x_{1}=l_{1}$, that fullfills for a scalar field

$$
\begin{aligned}
& z_{1^{\prime}}=x l_{1}-(3 x-U) \frac{z_{1}}{x}-\frac{k^{2}}{6 a^{2}} \frac{l_{1}}{x} s^{2}, \\
& l_{1^{\prime}}=6 x z_{1}-l_{1} \\
& x_{2^{\prime}}=3 x_{2}\left(\frac{\pi}{2}-1\right)+6\left(x z_{2}+U z_{1}\right)-3\left(2 x^{2}+1-\pi\right) l_{1}-x_{2}, \\
& z_{2^{\prime}}=3 z_{2}\left(\frac{\pi}{2}-1\right)+2 U l_{1}+4 x_{2} x-\left(\frac{k^{2}}{a^{2}}+m^{2}\right) s^{2} z_{1}
\end{aligned}
$$

The density contrast is a parameter used in cosmology while talking about structure formation to indicate if there exists local accumulation in the matter density. It is believed that after inflation even the universe was almost uniform,some regions were more dense than others with very big density contrast. As the universe expanded the gravitationally connected masses grew until they began to collapse, giving birth to galaxies, clusters, superclusters, etc...

Is common to define the density contrast as $\delta=\frac{\delta \rho}{\rho}$.

Taking into account our dimensionless variables we have an expression for the density contrast

$$
\frac{\delta \rho}{\rho}=\frac{2\left[x\left(z_{2}-x l_{1}\right)-U z_{1}\right]}{x^{2}+u^{2}}
$$

From the above equations we can notice that the unknown variables in our system are $\psi, \delta \Phi$ and $\rho$. If we know $\psi$ and $\delta \Phi, \rho$ can be calculated if we know the solutions to our dynamical system.

We now study the spherical collapse model with the SFDM paradigm following the classic formalism [??]. The spherical collapse model is a simple, but fundamental tool for understanding the growth of fluctuations in the Universe. This model considers that the formation of gravitational structures in the Universe can be described by the evolution of an overdense spherical region, this region decouples from the general expansion of the background Universe, slows down, reaches a maximum radius (this moment is called turn-around) and eventually collapses, virializes and stabilizes in a finite region.

We consider a spherical perturbation in scalar field dark matter field with a top-hat density profile. This means that the density of dark matter inside this region is spatially constant. In agreement with the spherical collapse formalism, the equations governing the evolution of this region ( cluster) are given by the Raychauduri equation and by the Klein-Gordon equation respectively:

$$
\frac{\ddot{R}}{R}=-\frac{\kappa^{2}}{6}\left(\rho_{\Phi_{c}}+3 P_{\Phi_{c}}\right)
$$

$$
\ddot{\Phi}_{c}+3 \frac{\dot{R}}{R} \dot{\Phi}_{c}+\frac{d V\left(\Phi_{c}\right)}{d \Phi_{c}}=0
$$

being $R$ a local scale factor, $\Phi_{c}, \rho_{\Phi_{c}}$ and $P_{\Phi_{c}}$ are the scalar field, the density and the pressure scalar inside cluster respectively. We set $\Phi_{c}=\Phi+\delta_{\Phi}\left(R_{i}, t\right)$, where $\Phi=\Phi(t)$ is the background scalar field and $\delta \Phi$ is a small perturbation.In this case $\delta_{\Phi}$ is independent of the spatial coordinates, this means that the perturbation is homogeneous inside the cluster (top-hat density profile). Therefore, the system of equations for the cluster are

$$
\begin{aligned}
& \frac{\ddot{R}}{R}=-\frac{\kappa^{2}}{6}\left(2 \dot{\Phi}^{2}+4 \dot{\Phi} \dot{\delta} \Phi+2 \dot{\delta} \Phi^{2}\right)-2 V(\Phi)-2 \delta \Phi \frac{d V(\Phi)}{d \Phi}, \\
& \ddot{\delta} \Phi=-3 \frac{\dot{R}}{R} \dot{\delta} \Phi-3 \dot{\Phi}\left(\frac{\dot{R}}{R}-\frac{\dot{a}}{a}\right)-\delta \Phi \frac{d^{2} V(\Phi)}{d \Phi^{2}} .
\end{aligned}
$$

The radiation field has not been included in the dynamics of the cluster because the radiation perturbations have no growing modes that affect the structure formation. Also, we have not included the baryonic perturbations because we assume they are accreated by the gravitational potential of collapsed structure of dark matter. On the other hand, the perturbations of cosmological constant are not important on small scales Following, we can define the non linear density contrast of dark matter at any time

$$
\delta \equiv\left(\rho_{\Phi}-\rho_{\Phi}\right) / \rho_{\Phi}=\delta \rho_{\Phi} / \rho_{\Phi},
$$

where $\delta \rho_{\Phi} \equiv \rho_{\Phi}-\rho_{\Phi}$ is given by

$$
\delta \rho_{\Phi}=\frac{1}{2} \dot{\delta} \Phi^{2}+\dot{\Phi} \dot{\delta} \Phi+\frac{d V(\Phi)}{d \Phi} \delta \Phi,
$$

in addition, we define $\delta P_{\Phi}$ as

$$
\delta P_{\Phi}=\frac{1}{2} \dot{\delta} \Phi^{2}+\dot{\Phi} \dot{\delta} \Phi-\frac{d V(\Phi)}{d \Phi} \delta \Phi .
$$

We suppose that the cluster evolves with the background Universe, however due to overdense of the cluster, it decouples slowing down of the general expansion of the background Universe. Following, the cluster reaches a point of maximum radius $R_{\text {max }}$, called turn-around (ta). After, the cluster continues its evolution and it begins to collapse to a singularity, however this is not a physical behavior because during the collapse our assumption of radial infall stops being worth. Instead of this, we suppose that the cluster collapses and dynamical equilibrium is reached. The radius of the system reaches a finite size and a bound structure is formed.In order to compute the virial radius $R_{v i r}$ of the bound structure of SFDM we study two cases. First we assume energy conservation between turn-around and virialization time. In this case, the equilibrium conditions reads 


$$
\begin{aligned}
\left.E_{T o t}\right|_{t a} & =\left.E_{\text {Tot }}\right|_{v i r} \\
\left.V\right|_{t a} & =\left.(T+V)\right|_{v i r}
\end{aligned}
$$

where $T$ is the kinetic energy and $V$ is the potential energy of the scalar field.

Now, we compute the potential energy for the scalar field.In order to do so, we calculate the gravitational potential $\psi$ for any cosmological component in a spherical shell as [32]

$$
\psi(r)=-\frac{\kappa^{2}}{4}(1+3 w) \rho\left(R^{2}-\frac{r^{2}}{3}\right)
$$

Obtaining dimensionless evolutions equations for the background universe and the cluster. In order to do, we define new dimensionless variable given by:

$$
\begin{gathered}
x \equiv \frac{\kappa}{\sqrt{6}} \frac{\dot{\Phi}}{H}, u \equiv \frac{\kappa}{\sqrt{3}} \frac{\sqrt{V}}{H}, \\
z \equiv \frac{\kappa}{\sqrt{3}} \frac{\sqrt{\rho_{z}}}{H}, v \equiv \frac{\kappa}{\sqrt{3}} \frac{\sqrt{\rho_{v}}}{H}, \\
b \equiv \frac{\kappa}{\sqrt{3}} \frac{\sqrt{\rho_{b}}}{H}, l \equiv \frac{\kappa}{\sqrt{3}} \frac{\sqrt{\rho_{\Lambda}}}{H} .
\end{gathered}
$$

we take the scalar potential $V(\Phi)=\frac{1}{2} m^{2} \Phi^{2}$, the evolution equations for the background are transformed into

$$
\begin{aligned}
x^{\prime} & =-3 x-s u+\frac{3}{2} \Pi x \\
u^{\prime} & =s x+\frac{3}{2} \Pi u \\
b^{\prime} & =\frac{3}{2}(\Pi-1) b \\
z^{\prime} & =\frac{3}{2}\left(\Pi-\frac{4}{3}\right) z \\
v^{\prime} & =\frac{3}{2}\left(\Pi-\frac{4}{3}\right) v \\
l^{\prime} & =\frac{3}{2} \Pi l \\
s^{\prime} & =s_{0} s^{-k}
\end{aligned}
$$

where the function $\Pi$ reads

$$
\Pi \equiv-\frac{2}{3} \frac{\dot{H}}{H^{2}}=2 x^{2}+b^{2}+\frac{4}{3} z^{2}+\frac{4}{3} v^{2}
$$

The variable $\mathrm{s}$ is a control parameter for the dynamics of $H$ whose exponent $k \leq 0$. With these variables, the density parameters $\Omega_{i}$ for each component $i$ can be written as

$$
\begin{aligned}
& \Omega_{D M}=x^{2}+u^{2}, \\
& \Omega_{\text {rad }}=z^{2}, \\
& \Omega_{\text {nеи }}=v^{2}, \\
& \Omega_{\text {bar }}=b^{2}, \\
& \Omega_{\Lambda}=l^{2} .
\end{aligned}
$$

With these new variables, we can write the equation of state of the scalar field as

$$
<w_{\Phi}>=<\frac{x^{2}-u^{2}}{\Omega_{D M}}>
$$

Following, the system of equations for the cluster with the square scalar potential are given by

$$
\begin{aligned}
& \frac{\ddot{R}}{R}=-\frac{\kappa^{2}}{6}\left(2 \dot{\Phi}^{2}+4 \dot{\Phi} \dot{\delta}_{\Phi}+2 \dot{\delta}_{\Phi}{ }^{2}\right)-2 m^{2} \Phi^{2}-2 \delta_{\Phi} m^{2} \Phi \\
& \ddot{\delta_{\Phi}}=-3 \frac{\dot{R}}{R} \dot{\delta}_{\Phi}-3 \dot{\Phi}\left(\frac{\dot{R}}{R}-\frac{\dot{a}}{a}\right)-m^{2} \delta_{\Phi} .
\end{aligned}
$$

The above system of equations (106) can be rewritten as a set of four coupled first-order differential equations using the next dimensionless variables:

$$
r \equiv \frac{\dot{R}}{m}, y \equiv \frac{\kappa}{\sqrt{6}} \frac{\dot{\delta_{\Phi}}}{H}, v \equiv \frac{\kappa}{\sqrt{6}} \frac{m \delta_{\Phi}}{H} .
$$

Finally the dimensionless system for the cluster is given by

$$
\begin{aligned}
& R^{\prime}=s r, \\
& r^{\prime}=\frac{R}{s}\left(-2 x^{2}-4 x y-2 y^{2}-u^{2}+2 u v\right), \\
& y^{\prime}=-3 x\left(\frac{r}{R} s-1\right)-s v-3 \frac{r}{R} s y+\frac{3}{2} \Pi y, \\
& v^{\prime}=s y+\frac{3}{2} \Pi v .
\end{aligned}
$$

The non linear density contrast can be written with the background and cluster dimensionless variables as

$$
\delta=\frac{y^{2}+2 u v}{\Omega_{D M}}
$$

\section{NUMERICAL RESULTS}

In this section we analyse both, the physics and dynamics of the results thrown by the dynamical system.We solve the system of equation of the background numerically(6) with a 
four order Adams-Bashforth-Moulton (ABM) method. We take as initial conditions the best estimations from 5-year WMAP values to $\Omega_{\Lambda}^{(0)}=0.721, \quad \Omega_{D M}^{(0)}=0.233$, $\Omega_{b}^{(0)}=0.0454, \Omega_{r}^{(0)}=0.0004, \quad \Omega_{v}^{(0)}=0.0002$ and the exponent $k=0$, where the superscript (0) is todays value of the density parameters. Fig. (1) shows the numerical evolution of the density parameters with our model. At the beginning, the radiation dominates the evolution of the Universe. After,the Universe has an epoch where the radiation is equal to dark matter $\left(a_{e q}\right)$, then dark matter begins to dominate the evolution. The recombination era in SFDM model is at $z: 1000$. Finally, the cosmological constant dominates the dynamics of the Universe. The behavior is exactly the same as in the $\Lambda \mathrm{CDM}$ model.

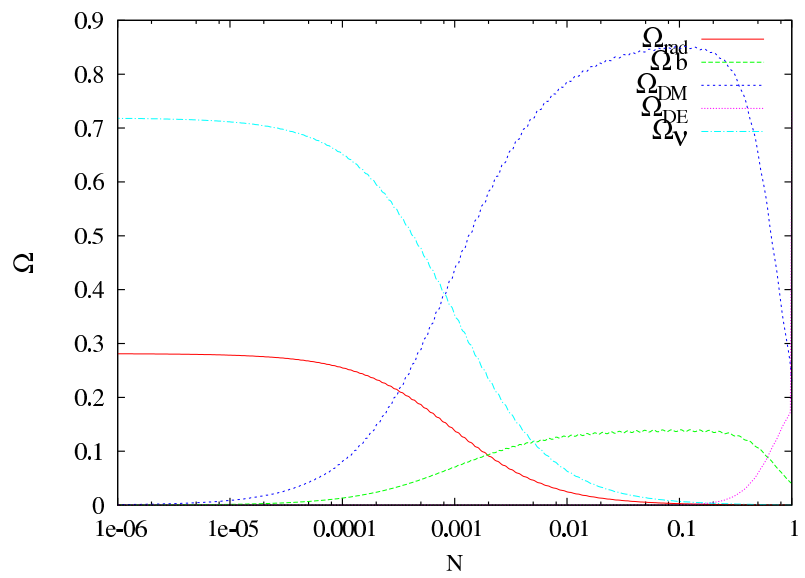

Fig. (8). Densities. We assume the Universe contains a real scalar field $\Phi$ as dark matter,radiation,neutrinos $v$, baryons b and a cosmological constant $\lambda$ as dark energy.

Once we have solved the evolution of the background,we can now obtain a numerical solution for the general system of equations. Firstly we study the evolution of the gravitational potential $\psi$ that perturbs the metric, as well as the perturbations over the scalar field that appear in KleinGordon's equation.

As initial conditions over $\psi$ we notice that in the matter dominant era ( $z: 1000$ ), the linearized Einstein equations can be solved analytically to obtain a solution of the form $\psi \propto t^{8 / 3}$ which implies that $\dot{\psi}$ takes an approximate value of zero during this epoch for all the values of $\mathrm{k}$, i.e, $\dot{\psi}=0$.

For the conditions over the scalar field the idea was to suppose that during this epoch, the perturbations were very small compared to other quantities like $\psi$, in fact in the code the best estimation for the gravitational potential was $\psi=1.0 \times 10^{-4}$. This implies that initially we can treat the scalar field as homogeneous.From this,we proposed $\delta \Phi$ and $\delta \dot{\Phi}=0$.

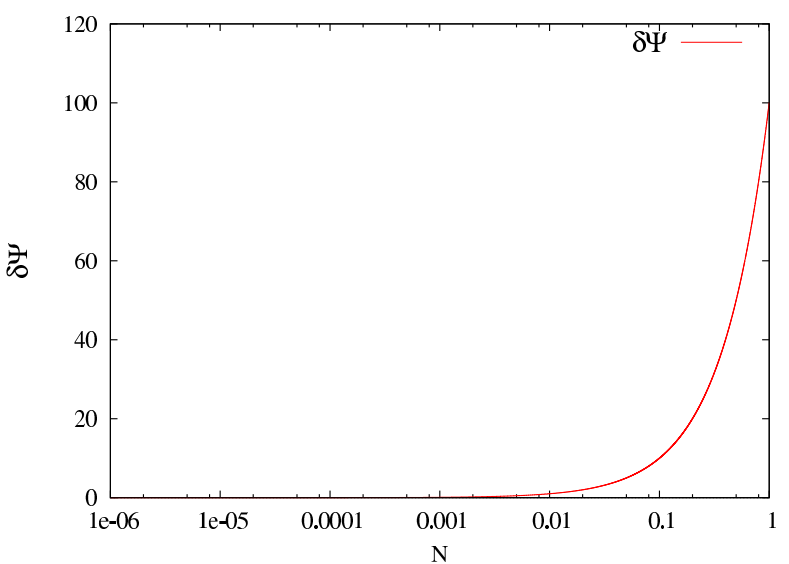

Fig. (9). Evolution of the gravitational potential.

From the figure we can see that the gravitational potential grows for $a \approx 1 \times 10^{-3}$, meaning that the fluctuations relate directly to fluctuations in the mass. As the perturbations grow, the gravitational potential grows,making it easier for gravitational collapse to occur.Now, if we want to study the power spectrum at large scales, we have to remember that this is valid when we work in the range $0.015 h M p c^{-1}<k<1 h M p c^{-1}$. In our system we took $k \approx 0.3 h M p c^{-1}$.

Finally as it was mentioned in [30] if we hope for this scalar field to form a BEC (Bose-Einstein Condensate),we then have an upper limit for the mass $m_{\Phi}$ of the scalar field, $m_{\Phi}<10^{-17} \mathrm{eV}$. We took $1 \times 10^{-23} \mathrm{eV}$.

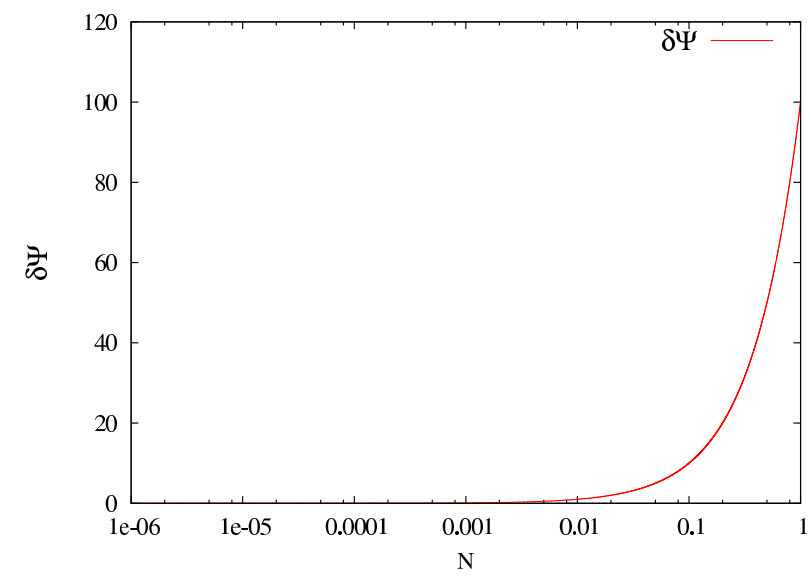

Fig. (10). Evolution of the perturbations over the scalar field.

For the perturbations on the scalar field we see that initially the perturbations to $\delta \Phi$ are nearly zero. Once it begins to dominate, the perturbations grow to scales not greater than $1 \times 10^{-38}$ meaning that the perturbations on the scalar field $\Phi$ can be neglected at first order, because of being so small without affecting in great way the evolution of other quantities. 


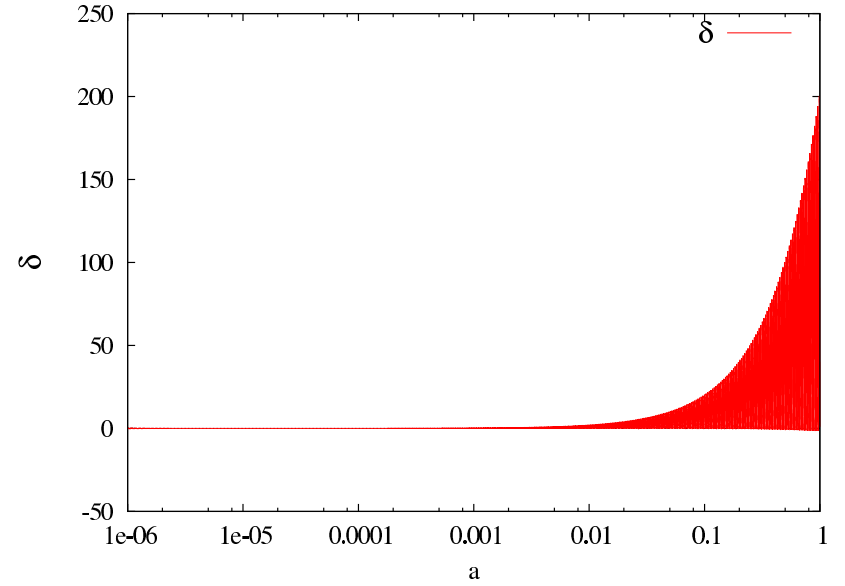

Fig. (11). Evolution of the density contrast $\delta$ for $k=0.3 h M p c^{-1} \Rightarrow \lambda=30 h^{-1} M p c$.

Now we have all the necessary ingredients to analyze one of the principal quantities when talking about structure formation. We analyze the density contrast, given by equation (92). In the denominator we can appreciate the total density of dark matter $x^{2}+u^{2}$. The density profiles give us information about the mass distribution there is, which is directly related to the structure formation theory, which is one our main objectives.

In the figure we can see that with the initial conditions imposed in our code at the beginning, there is a fast growing in our characteristic scale, for galaxy clustering.Recently observations have taken us to very early epochs in the origin of the Universe, and have made us think that structure had already been formed,corresponding to $z \approx 7$.

Its clear from the figure that at $z \lesssim 10$ there already existed well defined perturbations in the energy density, which can contribute to the early formation of structure. Then, if clusters could be formed as early as this z's, this would imply that $\Phi^{2}$ as a model for dark matter could give an explanation for the characteristic masses that are being observed.

The density contrast $\delta$ evolves lineally,except in rare regions were $\leq 1$ and gravitational collapse occurs.Then implotion of our massive object occurs under the influence of a gravitational force. When this occurs we take the following initial conditions, in the past at $a_{i}=10^{-6}$ and the values for the kinetic and potential term of the scalar field obtained in the previous numerical run for the background. For the initial radius of the cluster we take $R=a_{i}, r=$ and $y=x$.

Fig. (12) plots $R$ as functions of $a$. Initially, $R$ evolves as the background however, at the time when $\dot{R}=0, R$ increases to a maximus, this epoch of maximus radius is called the turn-around point. In this case, the turn around point is when $a_{t a}: 3.510^{-4}$, this mean a $z: 2800$. After, $R$ begins to decrease until it collapses into a singularity. This

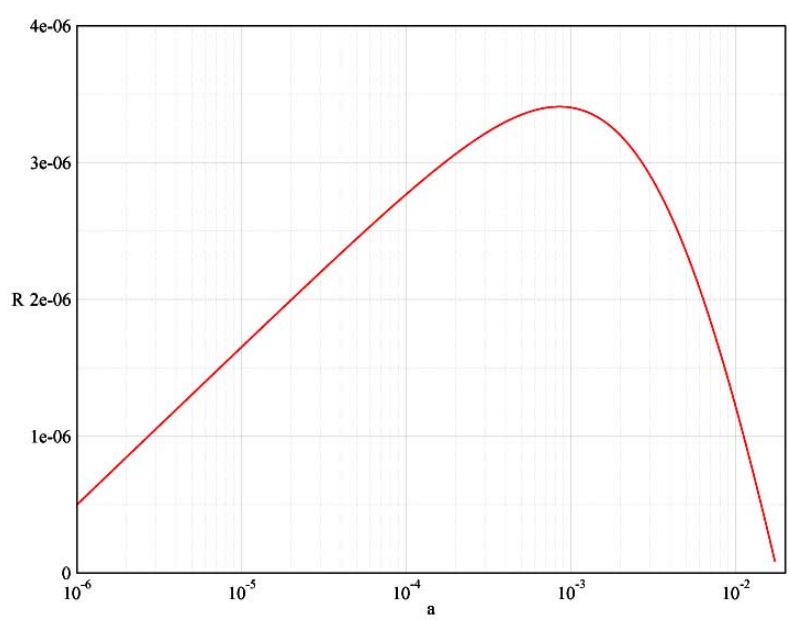

Fig. (12). Evolution of the radius of the cluster universe. Observe how the radius reaches a turn-around point and eventually collapses. The singularity is not real because the region can be formed in a virialized region.

predicted singularity is not physical because we assumed that dark matter is spherically distributed and collapses radially.In order to avoid this problem, we consider that the region collapses to a finite region and virializes according to the virial theorem. The value for $\delta_{t a}: 0.00$. This values is very small when we compare it with the $\Lambda \mathrm{CDM}$ paradigm, where $\delta_{\triangle C D M} \mathrm{I}_{t a}=4.5$. The small value for $\delta$ at turn-around suggest that the structure formation in our model is earlier than standard model. This means that the overdensities collapse faster and therefore we must have dark halos at early times.This is a prediction of scalar field dark matter and BEC. This result can be controversial however, there is evidence that suggests that dark halos could be formed at high redshift,some authors suggest that the downsizing in radiogalaxies is an sign of anti bottom up paradigm.

\section{CONCLUSIONS}

SFDM has provided to be an alternative model for the dark matter nature of the Universe. We have shown that the scalar field with a ultralight mass condensates very early in the Universe and generically form BEC's with a density profile which is very similar as that of the CDM model, but with a almost flat central density profile, as it seems to be in LSB and dwarf galaxies. This fact can be a crucial difference between both models. If the flat central density is no confirmed in galaxies, we can rule out the SFDM model, but if this observation is confirmed, this can be a point in favour of the SFDM model. We also show that the $1 / 2 m^{2} \Phi^{2}$ potential and the $V_{0}[\cosh (\kappa \lambda \Phi)-1]$ model are in fact the same. They have the same predictions and a spurious variable which determine the behavior of the model, given naturally the right expected cosmology and the same cosmology as the CDM model. This implies that the differences between both models, the CDM and SFDM ones, is in the non linear regime of perturbations, in the way they form galaxies and galaxy clusters, specially in the centre of galaxies where the SFDM model predicts a flat density 
profile. If the existence of supersymmetry is confirmed, the DM supersymmetric particles are observed by the detectors and they have the right mass, DM density and coupling constant, the SFDM can be ruled out. But, if these observations are not confirmed, the SFDM is an excellent alternative candidate to be the nature of the DM of the Universe.

We have investigated the evolution of the perturbations in the scalar field $\Phi$ around the universe.The cosmological limits here imposed are model dependent and therefore relay on the assumption of our theoretical model of structure that,even if in agreement with current data,may need further key ingredients and analysis to explain mysteries and inconsistencies such as dark energy.

With this important facts at hand, we conclude that the spectrums here presented for the different perturbations, depend deeply on what we assume for our initial conditions of the matter that now dominate the universe.we also studied the model of spherical collapse with an alternative model of dark matter, in this model the dark matter is a scalar field, we have shown that the collapse of an overdense region of scalar field dark matter collapses and virializes in a bound structure, but the formed structure is not consistent with real astrophysical parameters. However, further laboratory experiments will certainly test the cosmological results.

\section{ACKNOWLEDGEMENTS}

The numerical computations were carried out in the "Laboratorio de Super-Cómputo Astrofsico (LaSumA) del Cinvestav" and in the UNAM's cluster Kan-Balam. This work was partially supported by CONACyT México, under grants 49865-F and I0101/131/07 C-234/07, Instituto Avanzado de Cosmologia (IAC) collaboration.

\section{APPENDIX}

\section{DYNAMICAL SYSTEM REVIEW}

The theory of dynamical systems is used in the study of physical systems that evolve over time. It is assumed that the physical state of the system to an instant of time $t$ is described by an element $x$ of a space phase $X$, which can be of finite or infinite dimension. The evolution of the system is represented by a differential autonomous equation in $\mathrm{X}$, written symbolically as

$$
\frac{d \mathbf{x}}{d t}=\mathbf{f}(\mathbf{x}), \quad \mathbf{x} \varepsilon X
$$

where $\mathbf{f}: X \rightarrow X$.

The main step to get qualitative information on solutions is studying the flow of the equation in the vicinity of their critical points based on the Hartman-Grobman theorem, namely the study of its stability.

The essential idea is firstly find the fixed (or critical) points of the equation (110) which are given by $\mathbf{f}\left(\mathbf{x}_{\mathbf{c}}\right)=0$. Then linearized the differential equation at each critical point, that is, expanding about these points $\overrightarrow{\mathbf{x}}=\overrightarrow{\mathbf{x}}_{\mathbf{c}}+\delta \overrightarrow{\mathbf{x}}$ which yields to

$$
\delta \overrightarrow{\mathbf{x}}^{\prime}=\mathcal{M} \delta \overrightarrow{\mathbf{x}} .
$$

Therefore the general solutions for the linear perturbation evolution can be written as

$$
\delta \overrightarrow{\mathbf{x}}^{\prime}=\delta \overrightarrow{\mathbf{x}}_{\mathbf{0}} e^{\mathcal{N} \delta t}
$$

where $\mathcal{N}$ is the matrix composed of the eignvalues $m_{i}$ associated to $\mathcal{M}$.

The stability of the system (110) depends on the values of the eigenvalues: if the real part of all eigenvalues is negative, the fixed point is asymptotically stable, i.e., an attractor. All eigenvalues with positive real part make the fixed point asymptotically unstable (commonly called as source or repealer).

On the other hand, a saddle point happens when there exists a combinations of stable and unstable points. For a extended review seen, [33].

Then, we give a procedure for transforming equations (38) and (39), with an arbitrary potential, into a dynamical system. We define the dimensionless variables

$$
\begin{aligned}
& x \equiv \frac{\kappa}{\sqrt{6}} \frac{\dot{\Phi}}{H}, u \equiv \frac{\kappa}{\sqrt{3}} \frac{\sqrt{V}}{H}, \\
& z_{\gamma} \equiv \frac{\kappa}{\sqrt{3}} \frac{\sqrt{\rho_{\gamma}}}{H},
\end{aligned}
$$

Using the definitions given in (41), the evolution equations (38) transform into an autonomous system

$$
\begin{gathered}
x^{\prime}=-3 x+\frac{3}{2} \Pi x-\frac{\kappa}{\sqrt{6} H^{2}} V_{, \Phi} \\
u^{\prime}=\frac{3}{2} \Pi u+\frac{\kappa}{\sqrt{6} H^{2}} V_{, \Phi} \frac{x}{u} \\
z_{\gamma}{ }^{\prime}=\frac{3}{2}(\Pi-\gamma) z_{\gamma} \\
-\frac{H^{\prime}}{H}=\frac{3}{2}\left(2 x^{2}+\gamma z_{\gamma}^{2}\right) \equiv \frac{3}{2} \Pi
\end{gathered}
$$

This last equation (112d) can be written also as

$$
s^{\prime}=\frac{3}{2} \Pi s
$$

for the variable $s=c t e . / H$, and determines the evolution of the horizon. Here a prime denotes a derivative with respect to the e-folding number $N=\ln (a)$. The Friedmann equation (41) transforms into a constraint equation

$$
F=x^{2}+u^{2}+z_{\gamma}^{2}=1 \text {. }
$$


With these variables, the SFDM density can be written as

$$
\Omega_{D M}=x^{2}+u^{2},
$$

Observe that if we derive (114) with respect to $N$ and substitute system (112) into this, we obtain

$$
F^{\prime}=3(F-1) \Pi
$$

indicating that constraint (114) is compatible with system (112) for all scalar field potentials if the Friedmann equation is fulfilled.

Now we show that system (112) together with constraint (114) is completely integrable. To integrate system (112), first observe that we can substitute $3 / 2 \Pi$ from equation (113) into the rest of the equations. With this substitution equation (112c) can be integrated in terms of $s$ as

$$
z_{\gamma}=\sqrt{\Omega_{\gamma}^{(0)}} \operatorname{sexp}\left(-\frac{3}{2} \gamma N\right)
$$

where $\Omega_{\gamma}^{(0)}$ is an integrations constant. Now we multiply (112a) by $2 x$ and (112b) by $2 u$ and sum both equations. We obtain

$$
\left(x^{2}+u^{2}\right)^{\prime}=-6 x^{2}+2 \ln (s)^{\prime}\left(x^{2}+u^{2}\right)
$$

Now, we use constraint (114) and equation (116) into equation (117) to obtain

$$
6 x^{2}=2 \ln (s)^{\prime}-3 \gamma s^{2} \Omega_{\gamma}^{(0)} \exp (-3 \gamma N)
$$

But now we have to integrate equation (113) with all these results. If we substitute (118) and (116) into (112d) or (113) we obtain $0=0$, that means $S$ is an arbitrary variable and can be cast into the system as a control parameter. In other words, equations (112d) and (113) are actually identities, and not equations.

Thus, we set the variable $s$ from system (112) arbitrary in the system (112a), (112b) and (112c).

\section{REFERENCES}

[1] Nojiri S, Odinsov SD. Introduction to modified gravity and gravitational alternative for dark energy. Int J Geometric Method Phys 2007; 4: 115-19.

[2] Moffat JW. Scalar-tensor-vector gravity theory. J Cosmol Astropart Phys 2006; 3: 004.

[3] Guzmán FS, Matos T. Scalar fields as dark matter in spiral galaxies. Class Quantum Gravity 2000; 17: L9.

[4] Matos T, Guzmán FS. Scalar fields as dark matter in spiral galaxies. Comparison with Experiments. Astron Nachr 1999; 320: 97.

[5] Matos T, Ureña-López LA. Quintessence and scalar dark matter in the universe. Class Quantum Gravity 2000; 17: L75.

[6] Ji SU, Sin Sang-Jin. Phys Rev 1994; D50: 3655.
[7] Lee JW, Koh IG. Late-time phase transition and the galactic halo as a Bose liquid. II. The effect of visible matter. Phys Rev 1996; D53: 2236.

[8] Lee JW. Is dark matter a BEC or scalar field? J Korean Phys Soc 2009; 54: 2622. arXiv: 0801.1442.

[9] Matos T. Guzmán FS. On the Space-time of a Galaxy. Class Quantum Gravity 2001; 18: 5055 .

[10] Matos T., Guzmán F.S. Quintessence at Galactic Level? Ann Phys(Leipzig) 2000; 9: S133.

[11] Matos T. Ureña-López L.A. Scalar field dark matter, cross section and Planck-scale physics. Phys Lett B 2002; 538: 246.

[12] Ureña-López LA, Matos T, Becerril R. Inside oscillatons. Class Quantum Gravity 2002; 19: 6259.

[13] Turner MS. Coherent scalar-field oscillations in an expanding universe. Phys Rev 1983; D28: 1243.

[14] Seidel E. Suen W. Oscillating soliton stars. Phys Rev Lett 1991; 66: 1659 .

[15] Alcubierre M, Guzmán FS, Matos T, Nuñez D, Ureña-López LA, Wiedelhold P. Galactic collapse of scalar field dark matter. Class Quantum Gravity 2002; 19: 5017.

[16] Fukuyama T, Morikawa M, Tatekawa T. Cosmic structures via Bose Einstein condensation and its collapse. 2007. [arXiv: astro$\mathrm{ph} / 0705.3091]$

[17] Guzm'an FS, Ureña-López LA. Evolution of the Schr"odingerNewton system for a self-gravitating scalar field. Phys Rev 2004; D69: 124033.

[18] Guzmán FS. Ureña-López LA. Gravitational cooling of selfgravitating bose condensates. Astrophys J 2006; 645: 814

[19] Guzmán FS. Ureña-López LA. Newtonian collapse of scalar field dark matter. Phys Rev 2003; D68: 024023.

[20] Guzmán FS, Ureña-López LA. Evolution of the Schr"odingerNewton system for a self-gravitating scalar field. Phys Rev 2004; D69: 124033.

[21] Matos T, Guzmán FS, Núñez D. Spherical scalar field halo in galaxies. Phys Rev 2000; D62: 061301.

[22] de Blok WJG, Bosma A. High-resolution rotation curves of low surface brightness galaxies. Astron Astrophys 2002; 385: 816.

[23] de Blok WJG, Bosma A, McGaugh S. Simulating observations of dark matter dominated galaxies: towards the optimal halo profile. Mon Not R Astron Soc 2003; 340: 657.

[24] Simon JD, Bolatto AD, Leroy A, Blitz L. High-resolution measurements of the halos of four dark matter-dominated galaxies: deviations from a universal density profile. Astrophys J 2005; 621: 757.

[25] Sánchez-Salcedo FJ, Reyes-Iturbide J, Hernandez X. An extensive study of dynamical friction in dwarf galaxies: the role of stars, dark matter, halo profiles and MOND. Mon Not R Astron Soc 2006; 370: 1829 .

[26] Bernal A, Guzmán, FS. Scalar field dark matter: Nonspherical collapse and late-time behavior. Phys Rev 2006; D74: 103002.

[27] Cimatti A, Daddi E, Renzini P, et al. Old galaxies in the young Universe. Nature 2004; 430: 184.

[28] Hinshaw G. Five-Year Wilkinson Microwave Anisotropy Probe (WMAP) Observations: Data processing, sky maps, and basic results. Astrophys J Suppl 2009; 180: 225. [arXiv: 0803.0732].

[29] Ureña-López LA, Reyes-Ibarra MJ. On the dynamics of a quadratic scalar field potential. Int J Mod Phys 2009; D18: 621. [arXiv: astroph/0709.3996].

[30] Matos T. Ureña-López LA. Further analysis of a cosmological model with quintessence and scalar dark matter. Phys Rev 2001; D63: 63506. [arXiv: astro-ph/0006024].

[31] Bernal A, Matos T, Núñez D. Flat central density profiles from scalar field dark matter halo. Rev Mex Astron Astrophys 2008; 44 149.

[32] Landau L, Lifshitz E. The classical theory of fields. Oxford: Pergamon Prees 1975.

[33] Coley AA. Dynamical systems in cosmology. 1999. [arXiv: grqc/9910074].

Received: September 28, 2009

Revised: October 06, 2009

Accepted: October 06, 2009

(C) Matos et al.; Licensee Bentham Open.

This is an open access article licensed under the terms of the Creative Commons Attribution Non-Commercial License (http://creativecommons.org/licenses/by-nc/3.0/) which permits unrestricted, non-commercial use, distribution and reproduction in any medium, provided the work is properly cited. 